\title{
Biodata Mining of Differentially Expressed Genes between Acute Myocardial Infarction and Unstable Angina Based on Integrated Bioinformatics
}

\author{
Siyu Guo, ${ }^{1}$ Zhihong Huang, ${ }^{1}$ Xinkui Liu, ${ }^{1}$ Jingyuan Zhang, ${ }^{1}$ Peizhi Ye, ${ }^{2}$ Chao Wu, \\ Shan Lu, ${ }^{1}$ Shanshan Jia, ${ }^{1}$ Xiaomeng Zhang, ${ }^{1}$ Xiuping Chen, ${ }^{3}$ Miaomiao Wang, \\ and Jiarui $\mathrm{Wu}\left(\mathbb{1}^{1}\right.$ \\ ${ }^{1}$ Beijing University of Chinese Medicine, Beijing 100102, China \\ ${ }^{2}$ National Cancer Center, National Clinical Research Center for Cancer, Chinese Medicine Department of the Cancer Hospital of the \\ Chinese Academy of Medical Sciences and Peking Union Medical College, Beijing 100021, China \\ ${ }^{3}$ Institute of Chinese Medical Sciences, State Key Laboratory of Quality Research in Chinese Medicine, University of Macau, \\ Macau, China
}

Correspondence should be addressed to Jiarui Wu; exogamy@163.com

Received 14 February 2021; Revised 10 June 2021; Accepted 26 August 2021; Published 14 September 2021

Academic Editor: Stefano Pascarella

Copyright (c) 2021 Siyu Guo et al. This is an open access article distributed under the Creative Commons Attribution License, which permits unrestricted use, distribution, and reproduction in any medium, provided the original work is properly cited.

\begin{abstract}
Acute coronary syndrome (ACS) is a complex syndrome of clinical symptoms. In order to accurately diagnose the type of disease in ACS patients, this study is aimed at exploring the differentially expressed genes (DEGs) and biological pathways between acute myocardial infarction (AMI) and unstable angina (UA). The GSE29111 and GSE60993 datasets containing microarray data from AMI and UA patients were downloaded from the Gene Expression Omnibus (GEO) database. DEG analysis of these 2 datasets is performed using the "limma" package in R software. DEGs were also analyzed using protein-protein interaction (PPI), Molecular Complex Detection (MCODE) algorithm, Gene Ontology (GO), and Kyoto Encyclopedia of Genes and Genomes (KEGG) enrichment analysis. Correlation analysis and "cytoHubba" were used to analyze the hub genes. A total of 286 DEGs were obtained from GSE29111 and GSE60993, including 132 upregulated genes and 154 downregulated genes. Subsequent comprehensive analysis identified 20 key genes that may be related to the occurrence and development of AMI and UA and were involved in the inflammatory response, interaction of neuroactive ligand-receptor, calcium signaling pathway, inflammatory mediator regulation of TRP channels, viral protein interaction with cytokine and cytokine receptor, human cytomegalovirus infection, and cytokine-cytokine receptor interaction pathway. The integrated bioinformatical analysis could improve our understanding of DEGs between AMI and UA. The results of this study might provide a new perspective and reference for the early diagnosis and treatment of ACS.
\end{abstract}

\section{Introduction}

Acute coronary syndrome (ACS) is a complex syndrome of clinical symptoms, which is characterized by acute myocardial ischemia with severe coronary stenosis or occlusion caused by coronary plaque rupture and subsequent thrombosis, mainly including acute myocardial infarction (AMI) and unstable angina (UA) $[1,2]$. AMI can be divided into acute ST-segment elevation acute myocardial infarction (STEMI) and acute non-ST-segment elevation acute coronary syndrome (NSTEMI) due to the different stratification of early diagnosis and treatment [3]. According to the statistics of the American Heart Association (AHA) and the National Institutes of Health in 2019, there were about 633,000 patients with acute coronary syndrome in 2014, including about 609,000 AMI patients and about 24,000 UA patients [4]. In addition, a study showed that the incidence of AMI had been increasing in younger populations and decreasing 
in the elderly and that improvement in the in-hospital mortality of AMI may have reached a plateau in all age groups in Japan [5].

Based on clinical epidemiology, the mechanism of the relationship between UA and AMI was studied, and great progress has been made [6-9]. Studies have shown that some UA patients will develop into AMI if they are not treated properly $[10,11]$. Therefore, the identification of differential genes (DEGs) between UA and AMI is essential for the development of new targeted drugs to improve clinical efficacy.

With the development of high-throughput gene chip and transcriptome sequencing methods, more and more gene chip technologies are used in the research of cancer and cardiovascular diseases, which enriches the biological markers for early diagnosis, classification, and prognosis of diseases and also provides references for the targeted therapy of diseases [12-15]. Microarray technology is conducive to understanding gene association, location, expression, and linkage studies and is widely used to compare DEGs in patients with different diseases [16]. At present, although there have been some bioinformatics-based studies on the miRNA, DEGs, and mRNA of AMI, the role of DEGs between AMI and UA remains unclear [17, 18]. In this study, the microarray data containing both AMI and UA were downloaded from the GEO database, and the integrated bioinformatics method was adopted to screen the DEGs, protein-protein interaction (PPI), and related key pathways between AMI and UA. This research may provide new insights for understanding the functional regulation mechanism of DEGs between AMI and UA and help to develop relevant targeted therapies in further in-depth work. The flow chart of integrated bioinformatics research is shown in Figure 1.

\section{Materials and Methods}

2.1. Gene Expression Profile Data Collection. The GEO database (https://www.ncbi.nlm.nih.gov/gds/) developed and maintained by the National Center for Biotechnology Information (NCBI) not only collects and shares publicly available transcriptome data but also facilitates biomedical research [19]. In the current study, "Acute myocardial infarction" and "Unstable angina" were used as keywords to obtain gene expression microarray datasets related to AMI and UA. The criteria of the chip included are as follows: (1) the sample source of the dataset is human, (2) the dataset includes AMI and UA samples, and (3) there are no less than 20 samples per dataset.

2.2. Screening of DEGs. First, the gene expression dataset is translated into common gene symbols by using annotation information from the platform. The "limma" package of $\mathrm{R}$ software was utilized to screen the original dataset for expression normalization and differential expression analysis [20]. In addition, the actual situation determines whether the normalized data is $\log 2$ converted. The heatmap and volcano map show data results. The threshold of DEG screening was $|\log 2 \mathrm{FC}| \geq 1$ and $P<0.05$ (FC: fold change).
2.3. Protein Interaction Analysis and Module Analysis of $D E G s$. The interaction between proteins is crucial for understanding the metabolic and molecular mechanisms of diseases. The number of organisms covered by the STRING database (http://string-db.org, version: 11.0) is more than double to 5090 compared with the previous version. It collects, scores, and integrates all public sources of proteinprotein interaction information and supplements this information through computational predictions, including direct and indirect correlations between proteins [21]. In this study, DEGs were input into STRING, and the species was set as "Homo sapiens." In addition, the data with a confidence score greater than 0.7 was selected (low confidence: score $>0.15$, moderate confidence: score $>0.4$, and high confidence: score $>0.7)$. The results were imported into Cytoscape 3.7.1 (https://cytoscape.org/) to establish a protein-protein interaction (PPI) network model [22], and the MCODE (Molecular Complex Detection) plug-in was used for module analysis [23]. The parameters were set to the default values (degree cutoff $=2$, node score cutoff $=$ $0.2, K$-core $=2$, and Max.depth $=100)$. Then, the Maximal Clique Centrality (MCC) algorithm in "cytoHubba" was applied to screen the hub genes with high connectivity in PPI networks [24].

2.4. Enrichment Analysis of DEGs. GO provides a framework for describing the function of gene products in organisms, which can identify the biological characteristics of highthroughput genome and transcriptome data, including biological process (BP), cellular component (CC), and molecular function (MF) [25]. KEGG can be used in the functional interpretation and practical application of DEG information [26]. In order to reveal the biological significance of DEGs, DAVID v6.8 (https://david.ncifcrf.gov/), "clusterProfiler" [27], and "GOplot" packages [28] in R software were utilized for GO function and KEGG pathway enrichment analysis, and the results with FDR $<0.05$ were selected for analysis and visualization.

2.5. Expression Level Analysis and Correlation Analysis of Key DEGs. According to the MCC algorithm, 10 genes with the most stable and highest score in the network were selected as the key genes. The differences in hub gene expression between AMI and UA were shown using the boxplot tool in ImageGP (http://www.ehbio.com/ImageGP/). In addition, in order to better understand the key DEGs, the Pearson correlation coefficient was used for correlation analysis, and the "Corrplot" package in R software was used for visualization $[29,30]$.

\section{Results}

3.1. Screening of Differentially Expressed Genes. The present study finally included 2 datasets (GSE29111 and GSE60993), each of which contained AMI and UA samples, including 53 AMI samples and 25 UA samples (Table 1). DEG analysis of these 2 datasets is performed using the "limma" package in R software. A total of 242 differential genes were obtained from GSE29111, including 128 


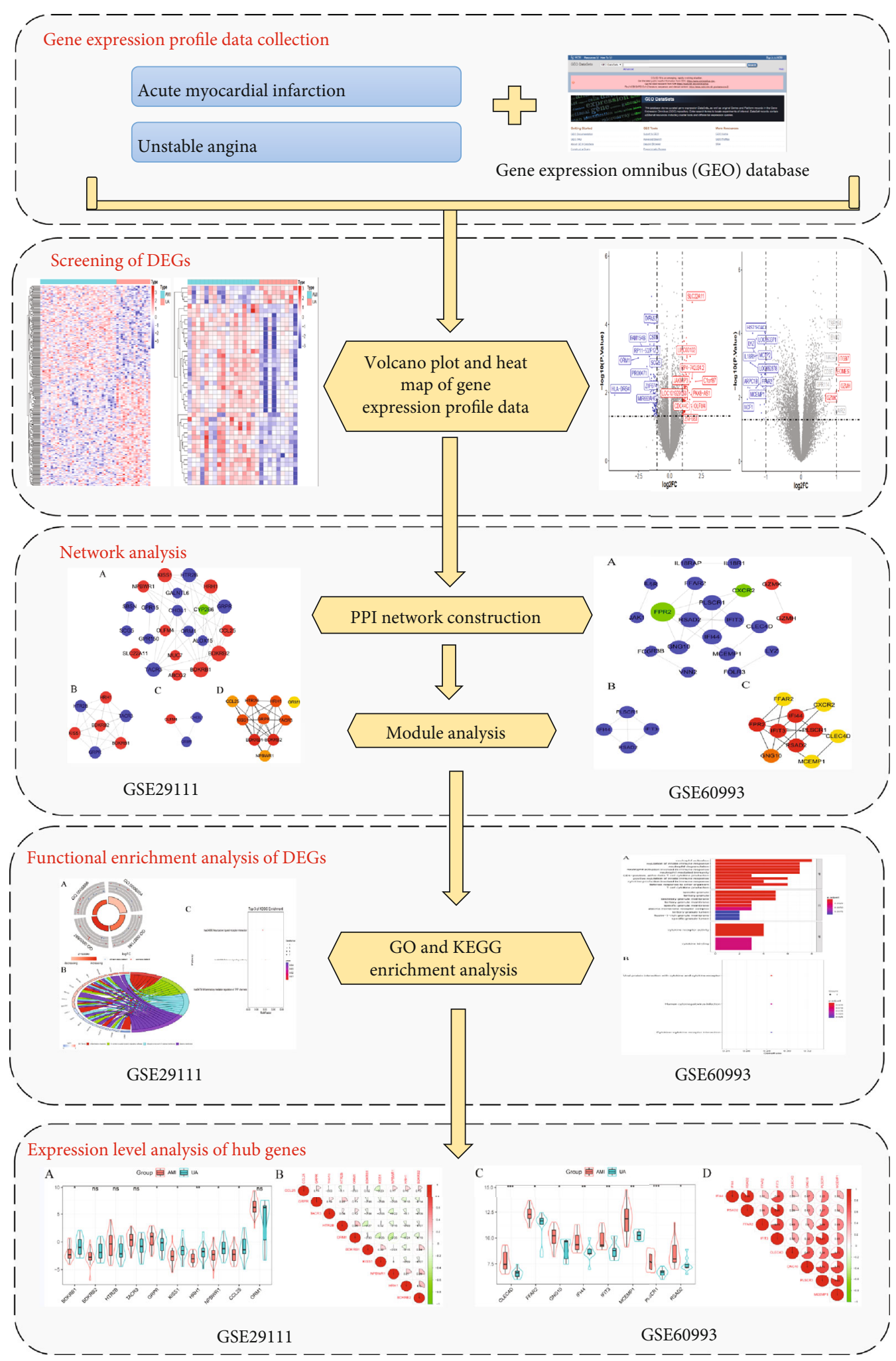

FiguRE 1: Integrated bioinformatics-based technical route for differential gene research between acute myocardial infarction and unstable angina pectoris.

upregulated genes and 114 downregulated genes (Supplementary Table 1). GSE60993 yielded 44 differentially expressed genes, including 4 upregulated genes and 40 downregulated genes (Supplementary Table 2). Figure 2 shows the DEG volcano plots and heatmaps of the included dataset. 
TABLE 1: Information of 2 microarray datasets of acute myocardial infarction and unstable angina.

\begin{tabular}{lcc}
\hline Dataset & Platform & Sample (AMI/UA) \\
\hline GSE29111 & GPL570 [HG-U133_Plus_2] Affymetrix Human Genome U133 Plus 2.0 Array & $36 / 16$ \\
GSE60993 & GPL6884 Illumina HumanWG-6 v3.0 expression beadchip & $17 / 9$ \\
\hline
\end{tabular}

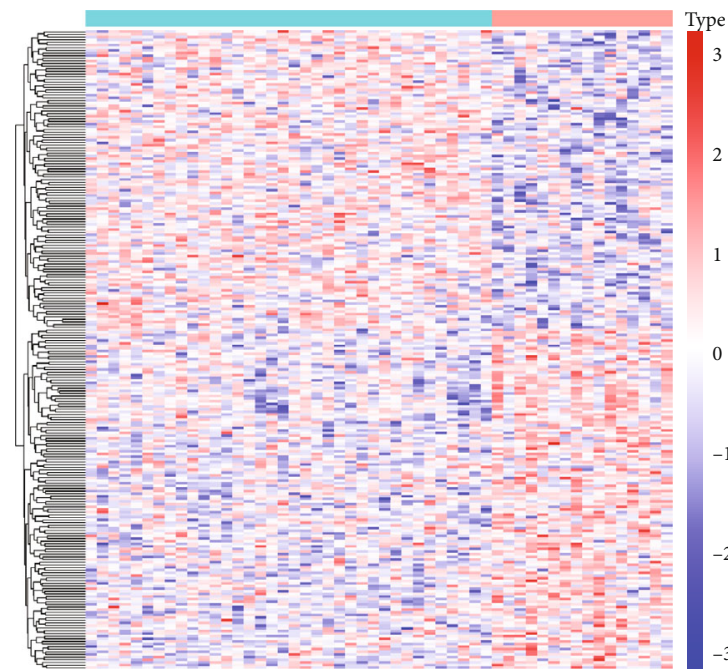

Type

AMI

UA

(a)

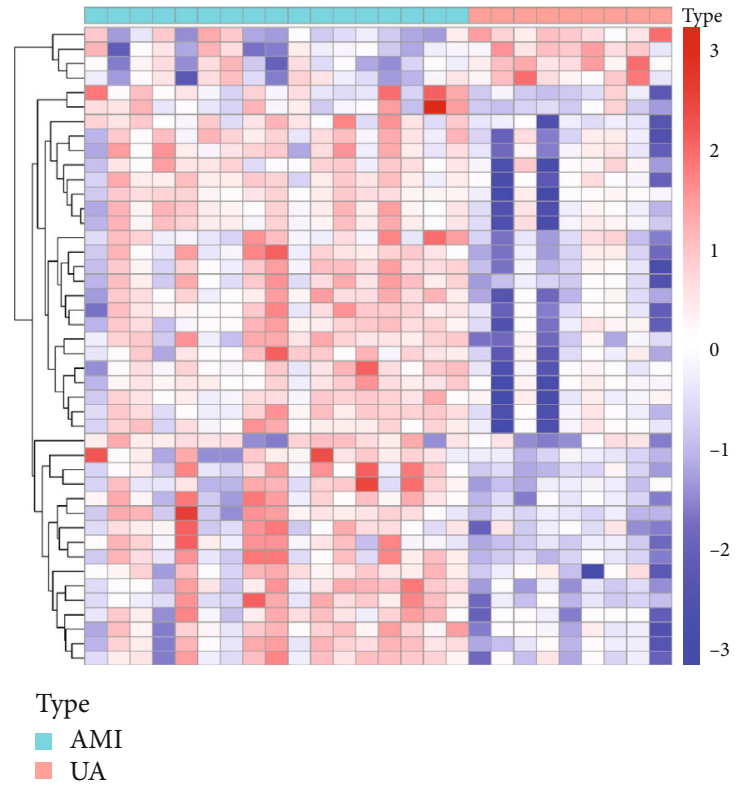

(c)

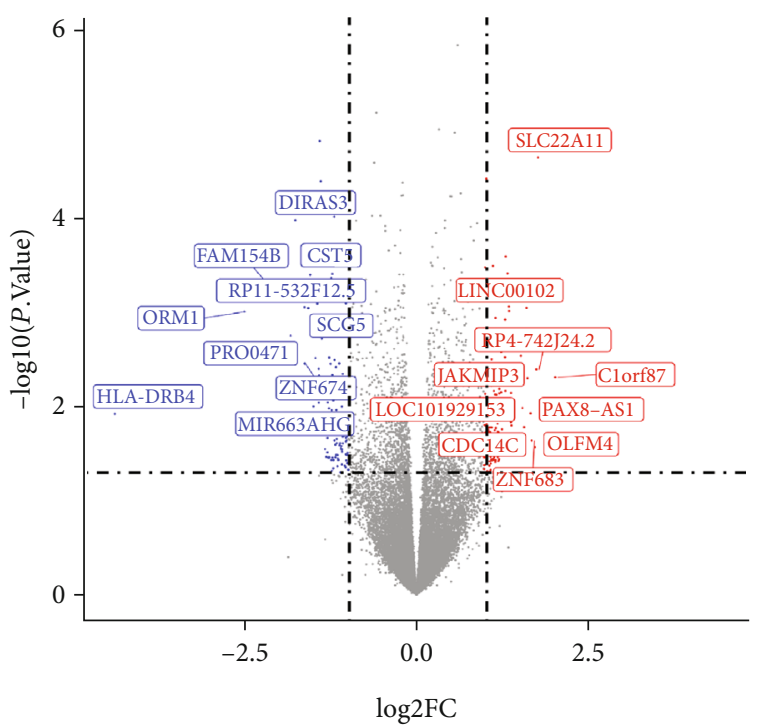

(b)

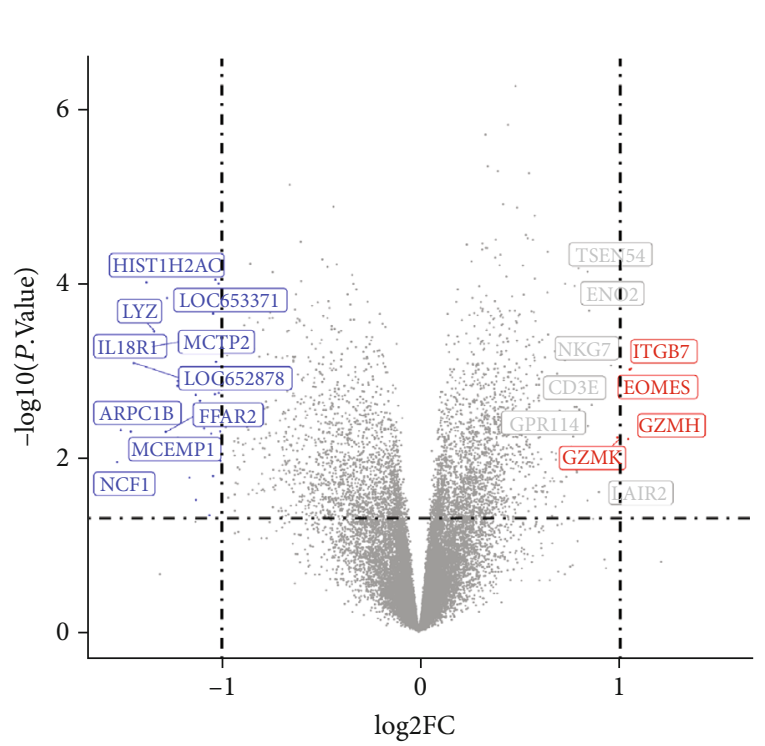

(d)

FIGURE 2: Heatmaps and volcano plots of differentially expressed genes between GSE29111 and GSE60993. (a) Heatmap of upregulated and downregulated genes of GSE29111. Each column represents a dataset, and each row represents a gene. Blue represents the downregulated gene, and red represents the upregulated gene. (b) Volcano plot of GSE29111. Blue represents the downregulated gene, red represents the upregulated gene, and gray represents the remaining differentially expressed genes. (c) Heatmap of upregulated and downregulated genes of GSE60993. (d) Volcano plot of GSE60993. 
3.2. Protein Interaction Analysis and Module Analysis of DEGs. The obtained DEGs were imported into the STRING database, and the data with a confidence score greater than 0.7 were selected. Cytoscape was used for network visualization analysis of these interacting DEGs. The PPI network of GSE29111 includes 22 nodes and 34 edges (Figure 3(a)). Using MCODE plug-in module analysis, a total of 2 significant modules were obtained (MCODE score $>3$ ) (Figures 3(b) and 3(c)). Module 1 contains 7 genes (4 upregulated and 3 downregulated), and module 2 contains 3 genes (1 upregulated and 2 downregulated). In addition, the PPI network is analyzed by the plug-in "cytoHubba" of Cytoscape. According to the MCC algorithm, 10 genes with the most stable and highest score in the network were selected as the hub genes: $\mathrm{B} 2$ bradykinin receptor (BDKRB2), histamine $\mathrm{H} 1$ receptor (HRH1), metastasis-suppressor KiSS-1 (KISS1), gastrin-releasing peptide receptor (GRPR), neuromedin-K receptor (TACR3), 5-hydroxytryptamine receptor 2B (HTR2B), C-C motif chemokine 25 (CCL25), neuropeptide $\mathrm{B} / \mathrm{W}$ receptor type 1 (NPBWR1), B1 bradykinin receptor (BDKRB1), and alpha-1-acid glycoprotein 1 (ORM1) (Table 2 and Figure 3(d)).

The PPI network of GSE60993 includes 20 nodes and 19 edges (Figure 4(a)). The MCODE plug-in was used for module analysis, and a significant module (score =4) was obtained. The genes included were downregulated genes (Figure 4(b)). The PPI network was analyzed by Cytoscape plug-in "cytoHubba." Based on the MCC algorithm, the 10 most stable and scored genes in the network were selected as the core genes: interferon-induced protein 44 (IFI44), free fatty acid receptor 2 (FFAR2), guanine nucleotide-binding protein $\mathrm{G}(\mathrm{I}) / \mathrm{G}(\mathrm{S}) / \mathrm{G}(\mathrm{O})$ subunit gamma-10 (GNG10), C-X$\mathrm{C}$ chemokine receptor type 2 (CXCR2), mast cell-expressed membrane protein 1 (MCEMP1), $\mathrm{N}$-formyl peptide receptor 2 (FPR2), C-type lectin domain family 4 member D (CLEC4D), interferon-induced protein with tetratricopeptide repeats 3 (IFIT3), radical S-adenosyl methionine domaincontaining protein 2 (RSAD2), and phospholipid scramblase 1 (PLSCR1) (Table 3 and Figure 4(c)).

3.3. Enrichment Analysis of DEGs. GO and KEGG pathway enrichment analysis was performed on PPI data of GSE29111 differentially expressed genes using DAVID and "GOplot" package in R software. According to FDR $<0.05$, 4 GO entries with significant enrichment were selected (Figures 5(a) and 5(b), Table 4). It contains 2 BP items and 2 CC items: inflammatory response, G-proteincoupled receptor signaling pathway, integral component of plasma membrane, and plasma membrane. According to FDR $<0.05,3$ significantly enriched KEGG pathways were screened: neuroactive ligand-receptor interaction, calcium signaling pathway, and inflammatory mediator regulation of TRP channels (Figure 5(c)).

In the current study, GO and KEGG pathway enrichment analysis was performed on PPI data of GSE60993 differentially expressed genes through the "clusterProfiler" package. According to $P$ adjust $<0.01$, a total of 52 significantly enriched GO entries were screened (Figure 6(a) and Table 5). There are $41 \mathrm{BP}$ items, mainly related to neutrophil activation, immune response, and inflammatory response; 9 CC items, primarily relevant to particles and plasma membrane; and $2 \mathrm{MF}$ items, basically associated with cytokine receptor activity and cytokine binding. According to $P$ adjust $<0.05,3$ significantly enriched KEGG pathways were screened: viral protein interaction with cytokine and cytokine receptor, human cytomegalovirus infection, and cytokine-cytokine receptor interaction (Figure 6(b)).

3.4. Expression Level and Correlation Analysis of Key DEGs. In order to better understand the key DEGs screened by GSE29111 and GSE60993, their expression levels and correlation analysis were performed. According to Pearson's correlation coefficient $(r)$ classification, the absolute values of $0-0.30,0.30-0.50,0.50-0.70$, and $0.70-1.00$ indicated "poor," "medium," "good," and "strong" correlations. In addition, " $r=0$ " means completely irrelevant and " $r=1.00$ " means completely relevant. The key DEGs screened by GSE29111, BDKRB1, BDKRB2, CCL25, HRH1, KISS1, and NPBWR1 were highly expressed in UA patients and lowly expressed in AMI patients. GRPR, TACR3, HTR2B, and ORM1 were lowly expressed in UA patients and highly expressed in AMI patients (Figure 7(a)). As shown in Figure $7(\mathrm{~b})$, the correlations between BDKRB1, BDKRB2, CCL25, HRH1, KISS1, NPBWR1, GRPR, TACR3, HTR2B, and ORM1 were poor or moderate.

The key DEGs screened by GSE60993, CLEC4D, FFAR2, GNG10, IFI44, IFIT3, MCEMP1, PLSCR1, and RSAD2 were highly expressed in UA patients and lowly expressed in AMI patients (Figure 7(c)). As shown in Figure 7(d), CLEC4D, FFAR2, GNG10, IFI44, IFIT3, MCEMP1, PLSCR1, and RSAD2 were positively correlated. Among them, PLSCR1 showed a strong positive correlation with MCEMP1 $(r=0.82)$. IFI44, RSAD2, and CLEC4D showed a poor positive correlation $(r=0.25)$.

\section{Discussion}

Many factors affect the occurrence and development of ACS, such as gender, fatigue, and frailty [31-33]. According to "China cardiovascular disease report 2018," the mortality rate of AMI showed an overall upward trend from 2002 to 2016 , and the mortality rate in rural areas was higher than that in urban areas many times. Although a lot of research has been conducted on the molecular mechanisms related to AMI and UA, the understanding of the differentially expressed genes between AMI and UA is insufficient. Therefore, it is still a challenge to diagnose the disease types of ACS patients quickly and accurately. Microarray analysis is a powerful tool for screening hub genes, which can effectively reduce candidate genes associated with multifactorial diseases and identify potential molecular mechanisms of disease and biomarkers for diagnosis and prognosis [34, 35]. In this study, we obtained the microarray datasets of AMI and UA patients from the GEO database to clarify their DEGs and candidate biomarker.

The integrated bioinformatics analysis method was performed to screen the potential differential genes related to AMI and UA. Through retrieval, 2 datasets (GSE29111 and 


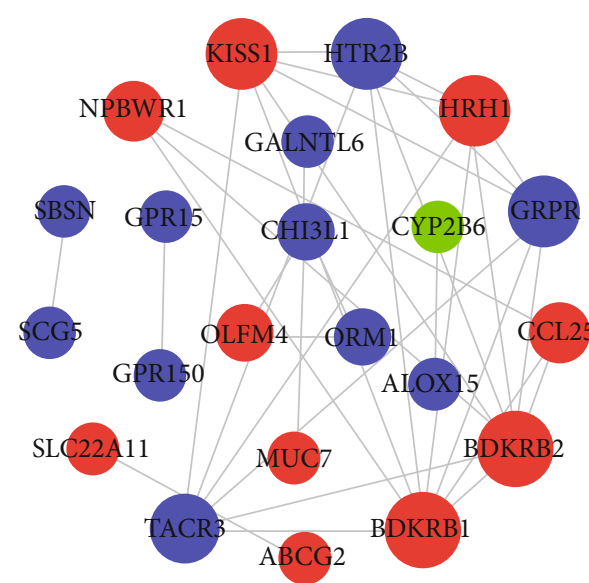

(a)

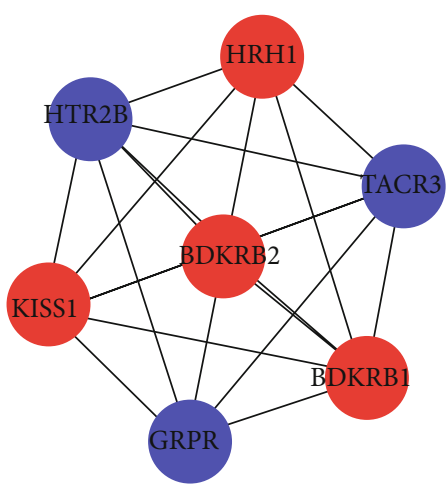

(b)

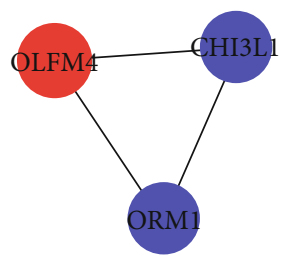

(c)

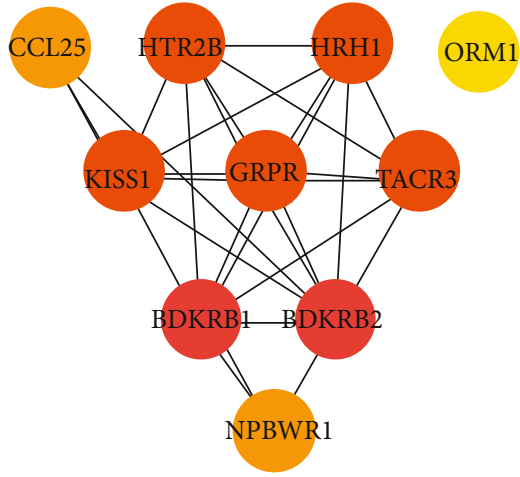

(d)

FIgURE 3: Protein-protein interaction (PPI) network and hub clustering modules of GSE29111. (a) The PPI network of DEGs. (b) Module 1 (MCODE score $=7)$. (c) Module $2($ MCODE score $=3)$. Blue represents the downregulated gene, and red represents the upregulated gene. The size of the node is proportional to the degree. (d) The connectivity map of 10 hub genes. From yellow to red, the darker the color, the higher the MCC score.

TABLE 2: Information of key genes in PPI network of GSE29111.

\begin{tabular}{lcccc}
\hline Gene & UniProt ID & Degree & Expression level & Module \\
\hline BDKRB2 & P30411 & 8 & Upregulation & Module 1 \\
HRH1 & P35367 & 6 & Upregulation & Module 1 \\
KISS1 & Q15726 & 6 & Upregulation & Module 1 \\
GRPR & P30550 & 6 & Downregulation & Module 1 \\
TACR3 & P29371 & 6 & Downregulation & Module 1 \\
HTR2B & P41595 & 6 & Downregulation & Module 1 \\
CCL25 & O15444 & 3 & Upregulation & - \\
NPBWR1 & P48145 & 3 & Upregulation & - \\
BDKRB1 & P46663 & 8 & Upregulation & Module 1 \\
ORM1 & P02763 & 2 & Downregulation & Module 1 \\
\hline
\end{tabular}

GSE60993) from the GEO database were included. Although datasets as GSE29111 and GSE60993 were already used and published by some authors, the main comparison is the DEGs between AMI and healthy controls. The difference is that this study used these two datasets to explore the DEGs between AMI and UA. A total of 242 DEGs were obtained from GSE29111, including 128 upregulated genes and 114 downregulated genes. There were 44 DEGs obtained from GSE60993, including 4 upregulated genes and 40 downregulated genes. According to the MCC algorithm, the 10 most stable and highest score genes in the PPI network were selected as core genes. The hub genes involved in GSE29111 included BDKRB1, BDKRB2, CCL25, HRH1, KISS1, NPBWR1, GRPR, TACR3, HTR2B, and ORM1. The core genes involved in GSE60993 include IFI44, FFAR2, GNG10, CXCR2, MCEMP1, FPR2, CLEC4D, IFIT3, RSAD2, and PLSCR1. These identified core genes act as a whole and may play an important role in the early diagnosis of ACS.

GO enrichment analysis showed that the DEGs screened from GSE29111 were mainly enriched in the inflammatory response, G-protein-coupled receptor signaling pathway, integral component of plasma membrane, and plasma membrane. The GO entries of the DEGs screened from the GSE60993 were mainly related to neutrophil activation, immune response, inflammatory response, particles, plasma membrane, cytokine receptor activity, and cytokine binding. Over the past decades, the critical role of immune inflammatory processes in the occurrence, development, and deterioration of many cardiovascular diseases has received 


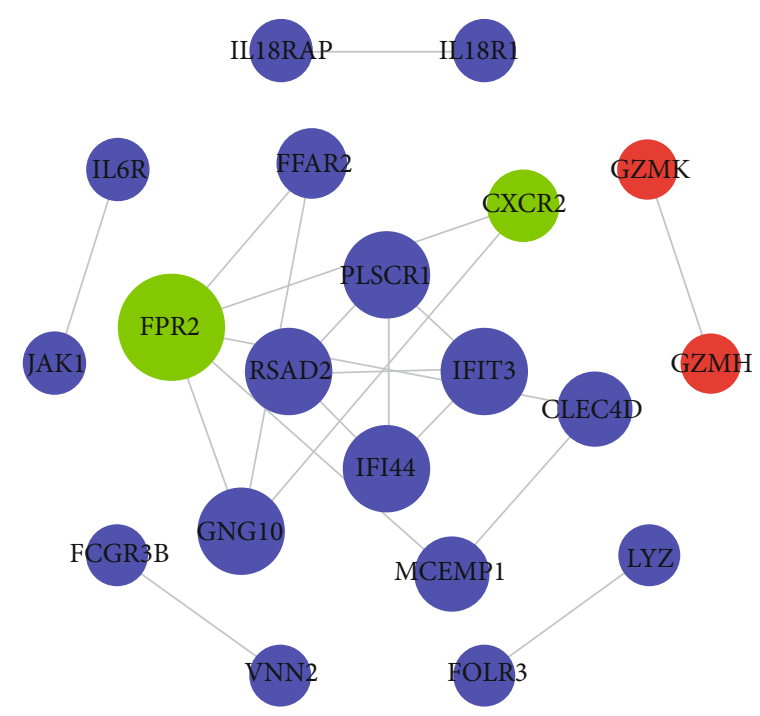

(a)

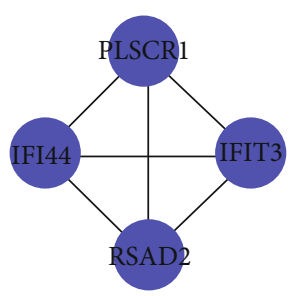

(b)

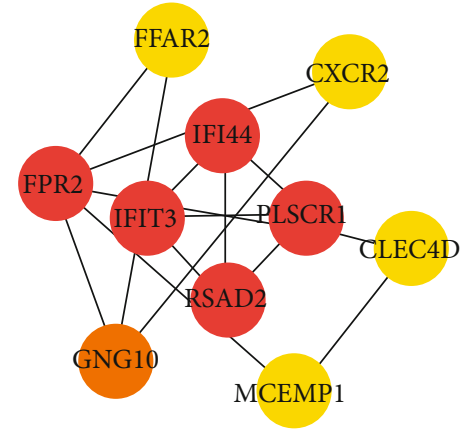

(c)

FIGURE 4: Protein-protein interaction (PPI) network and hub clustering modules of GSE60993. (a) The PPI network of DEGs. (b) Module 1 (MCODE score $=4)$. Blue represents the downregulated gene, and red represents the upregulated gene. The size of the node is proportional to the degree. (c) The connectivity map of 10 core genes. From yellow to red, the darker the color, the higher the MCC score.

TABLE 3: Information of key genes in protein interaction network of GSE60993.

\begin{tabular}{lcccc}
\hline Gene & UniProt ID & Degree & Expression level & Module \\
\hline IFI44 & Q8TCB0 & 3 & Downregulation & Module 1 \\
FFAR2 & O15552 & 2 & Downregulation & - \\
GNG10 & P50151 & 3 & Downregulation & - \\
CXCR2 & P25025 & 2 & - & - \\
MCEMP1 & Q8IX19 & 2 & Downregulation & - \\
FPR2 & P25090 & 5 & - & - \\
CLEC4D & Q8WXI8 & 2 & Downregulation & - \\
IFIT3 & O14879 & 3 & Downregulation & Module 1 \\
RSAD2 & Q8WXG1 & 3 & Downregulation & Module 1 \\
PLSCR1 & O15162 & 3 & Downregulation & Module 1 \\
\hline
\end{tabular}

increasing attention, such as atherosclerosis, myocardial infarction, heart failure, myocarditis, and vasculitis [36, 37]. At present, many biological, immunoregulatory, and antioxidant strategies have been proposed for heart protection [38]. Ong et al. summarized the inflammatory response after AMI; the results showed that the attack of acute myocardial ischemia can cause cell damage and death of different myocardial components [39]. Moreover, it triggers acute proinflammatory responses through a synergistic effect of multiple processes, which leads to the release of a variety of proinflammatory mediators and induces the recruitment of inflammatory cells to the infarction area [40]. In the animal model of AMI, the injured myocardial cells released interleukin-1 alpha (IL-1 $\alpha$ ), while interleukin-1 beta (IL$1 \beta$ ) increased after infarction [41]. The findings of our study suggest that the DEGs between AMI and UA are related to the inflammatory response, which provides a reference for their treatment.

The enrichment analysis of the KEGG pathway showed that the main enriched pathways of DEGs screened from the GSE29111 dataset were related to the interaction of neuroactive ligand-receptor (hsa04080), calcium signaling pathway (hsa04020), and inflammatory mediator regulation of TRP channels (hsa04750). Previous studies have shown that the nervous system regulation of cardiac activity has nothing to do with the physiological state of the heart [42]. However, the autonomic balance between sympathetic and parasympathetic systems plays an important role in the regulation of the cardiovascular system [43]. The facts show that the interruption of cardiac parasympathetic (vagus) activity is a common feature of various cardiovascular diseases including AMI [44]. In addition, some animal experiments have shown that increased vagus nerve activity has a protective effect on the heart of myocardial ische$\mathrm{mia} /$ reperfusion injury $[45,46]$. Wang et al. showed that in patients undergoing coronary artery bypass grafting, the cardioprotective effect of sevoflurane was related to the interaction of neuroactive ligand-receptor [47]. Chen et al. showed that the neuroactive ligand-receptor interaction pathway was closely related to human arrhythmogenic right ventricular cardiomyopathy [48]. Although the correlation between AMI and neuroactive ligand-receptor interaction has not been reported, this pathway may be a new mechanism of AMI, which is worth further study.

Calcium, as a common intracellular second messenger, is involved in regulating fertilization, gene transcription, secretion, and myocardial cells [49]. Ruiz-Meana et al. showed that calcium increased during myocardial infarction [50]. Garcia-Dorado et al. proposed to develop effective and reliable treatments to inhibit $\mathrm{Ca}^{2+}$-mediated myocardial cell 


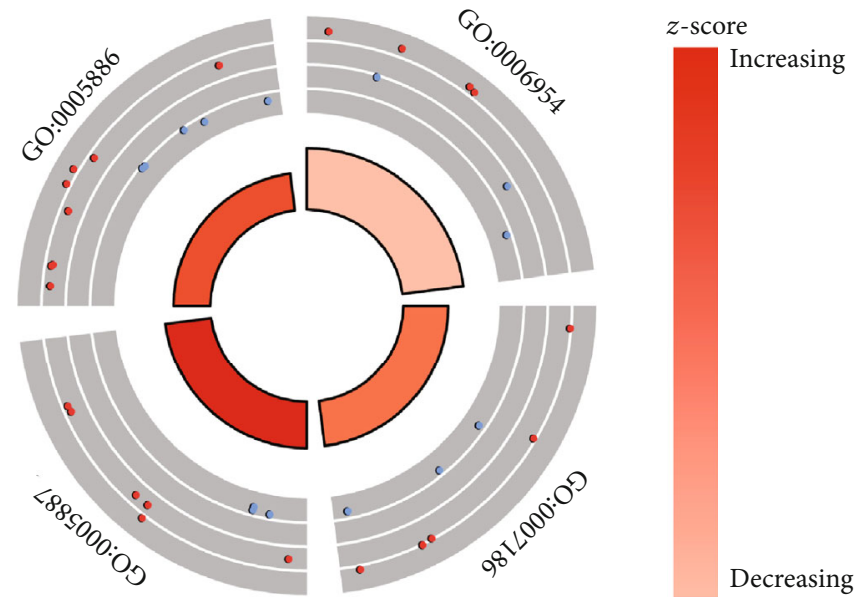

$\log \mathrm{FC}$

- Downregulated

- Upregulated

(a)

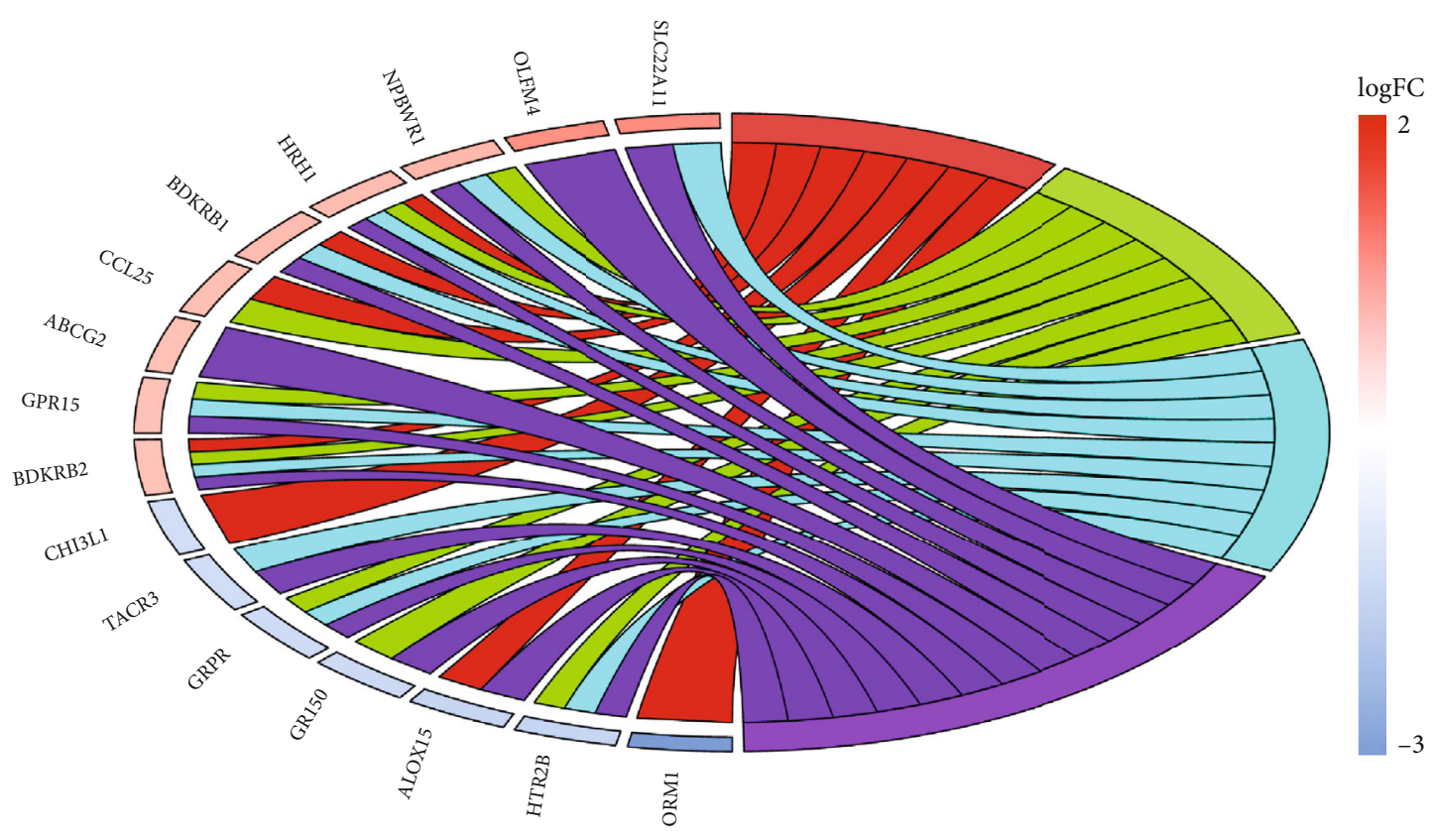

GO terms

Inflammatory response

G-protein coupled receptor signaling pathway
Integral component of plasma membrane

Plasma membrane

(b)

Figure 5: Continued. 


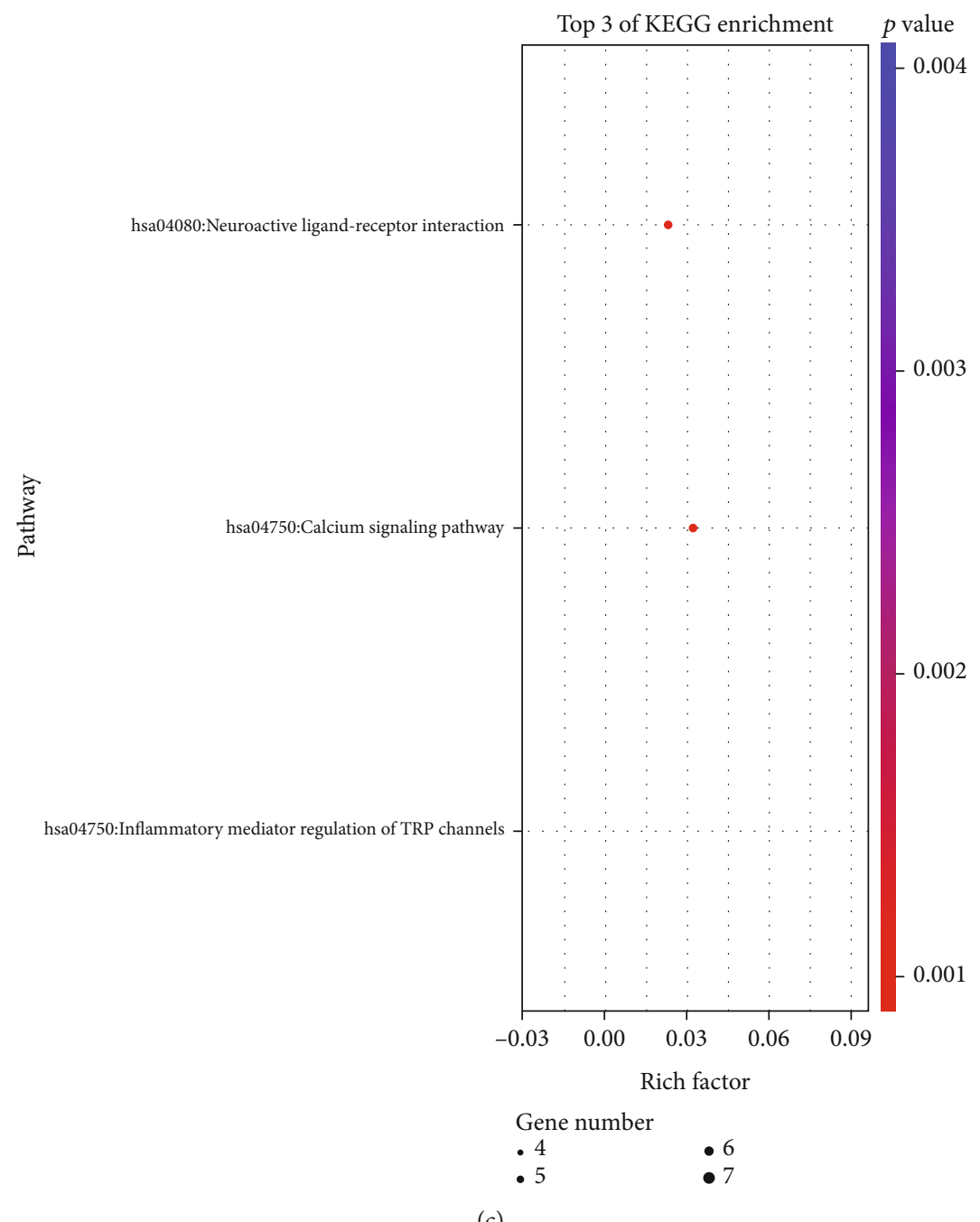

(c)

FIgURE 5: GO and KEGG pathway enrichment analysis of DEGs in GSE29111. (a) Circular plot for GO enrichment analysis of DEGs. The inner circle represents the $z$-score, which is the downregulation of enriched genes under a certain GO entry. The outer circle represents GO item ID. The points in the outer ring represent the distribution of downregulated genes involved in this item. (b) Chord plot for GO enrichment analysis of DEGs. The right side represents GO entries, and different colors represent different GO entries. The left side represents genes enriched in GO entries. (c) KEGG pathway enrichment analysis of DEGs. The horizontal axis represents the rich factor, and the vertical axis represents the KEGG pathway.

TABLE 4: Information on GO entries enriched by differentially expressed genes in GSE29111.

\begin{tabular}{lccc}
\hline Category & ID & Item & FDR \\
\hline BP & GO:0006954 & Inflammatory response & $6.67 E-04$ \\
BP & GO:0007186 & G-protein-coupled receptor signaling pathway & 0.004568 \\
CC & GO:0005887 & Integral component of plasma membrane & 0.003999 \\
CC & GO:0005886 & Plasma membrane & 0.012242 \\
\hline
\end{tabular}

death in patients with myocardial infarction, which has important therapeutic significance and potential clinical effects [51]. Consistent with our results, the calcium signaling pathway was found to be one of the most significant pathways. Recent studies have shown that the regulation of TRP channels mainly occurs at transcription, translation, and posttranslation levels and depends on ion balance, microbial ligands, cytokines, or reactive oxygen species (ROS). In fact, inflammatory transcription factors such as nuclear factor $-\kappa \mathrm{B}(\mathrm{NF}-\kappa \mathrm{B})$, signal transduction and transcription activation factor 3 (STAT3), and hypoxiainducible factor-1 (NIF-1) are all related to the increase of 


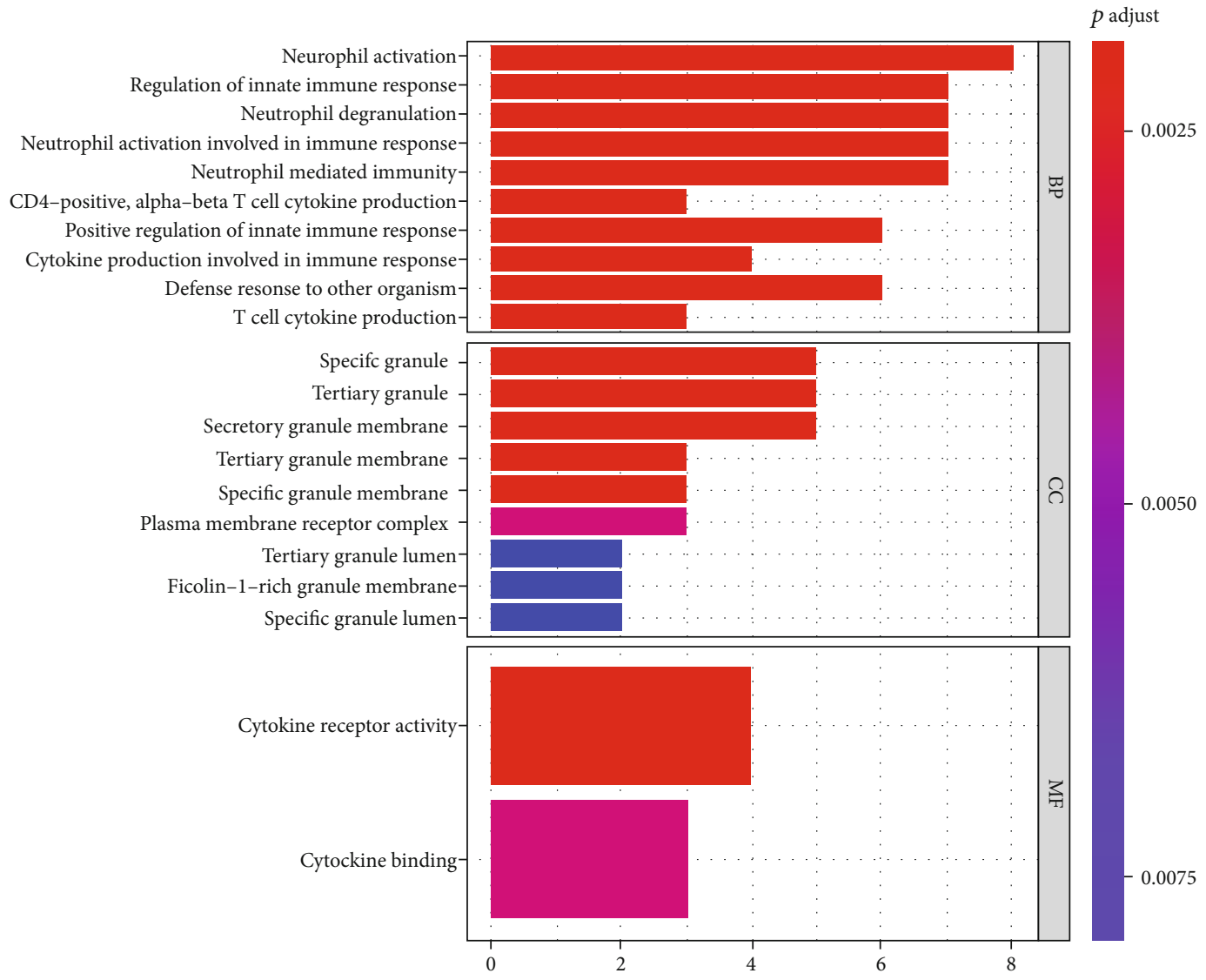

(a)

Figure 6: Continued. 


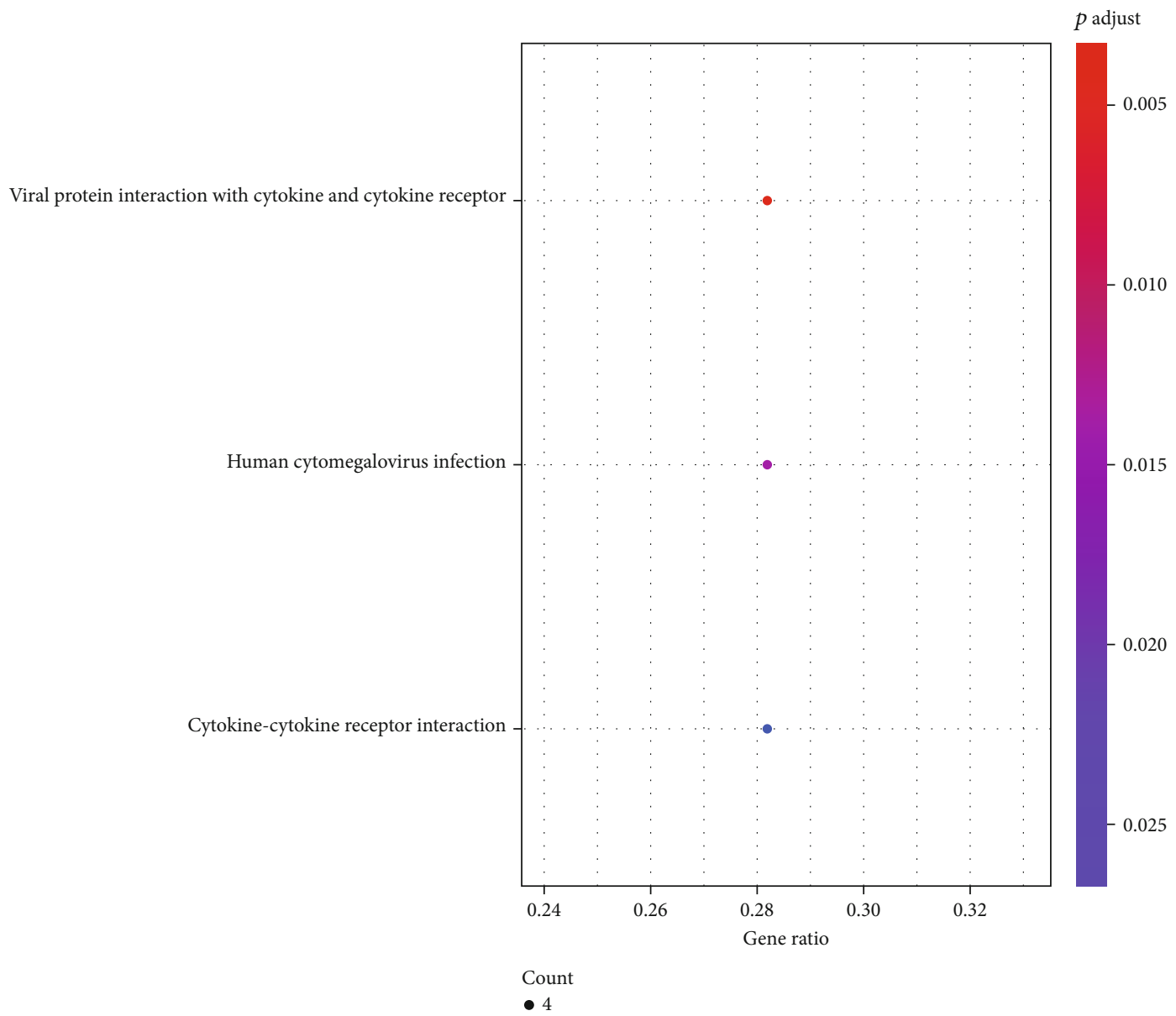

(b)

FIGURE 6: GO and KEGG pathway enrichment analysis of DEGs in GSE60993. (a) GO enrichment analysis of DEGs. (b) KEGG pathway enrichment analysis of DEGs. The horizontal axis represents gene ratio, which is the ratio of the number of genes associated with the pathway to the total number of DEGs. The vertical axis represents the KEGG pathway.

ROS and intracellular $\mathrm{Ca}^{2+}$ concentration, and these characteristics make TRP channels effectively regulate inflammation [52]. Although neuroactive ligand-receptor interaction, calcium signaling pathway, and inflammatory mediator regulation of TRP channels have little research on ACS, they may be new ways of occurrence and development of AMI and UA, which is worthy of further study.

In the current study, the core genes enriched in the neuroactive ligand-receptor interaction with the smallest FDR value were discussed and analyzed in detail. HRH1, NPBWR1, BDKRB2, HTR2B, TACR3, BDKRB1, and GRPR are 7 core genes enriched in the neuroactive ligand-receptor interaction. Histamine receptor $\mathrm{H} 1$ (HRH1) belongs to the G-protein-coupled receptor family. Its gene products are expressed in many tissues such as smooth muscle and neurons $[53,54]$. Bhuiyan et al. found that HRH1 is mainly expressed in the neurons of the solitary tract nucleus, and histamine in the solitary tract nucleus may play a role in regulating cardiovascular homeostasis by activating $\mathrm{H} 1$ receptors expressed in the neurons of the solitary tract nucleus [55]. One study showed that the activation of HRH1 drove the formation of atherosclerotic lesions by increasing the vascular permeability of low-density lipoprotein [56]. Neuropeptide W (NPW) and neuropeptide $\mathrm{B}$ (NPB) are 2 structural and functional regulatory peptides. They are highly expressed in some brain regions and peripheral tissues (such as the heart, kidney, and stomach), but their distribution in tissues is not similar $[57,58]$. In addition, they act on target tissues through neuropeptide $\mathrm{B} / \mathrm{W}$ receptor 1 (NPBWR1) and neuropeptide $\mathrm{B} / \mathrm{W}$ receptor 2 (NPBWR2). NPB activates NPBWR1, while NPW stimulates the 2 receptors with similar potency and has different binding affinities $[59,60]$. NPW increases the calcium flow into the cells by acting on NPBWR1 and plays a role in regulating vascular myofibrillar tension, so it may be involved in the development of hypertension [61]. In a rat model, the results suggest that central NPW30 can increase sympathetic outflow and affect cardiovascular function [62]. In the past few years, a large number of literatures have shown that B1 bradykinin receptor (BDKRB1) and $\mathrm{B} 2$ bradykinin receptor (BDKRB2) are involved in a variety of hemodynamics and metabolism [63]. Marketou et al. found that early use of selective BDKRB2 agonist in the treatment of AMI in mice could 
TABLE 5: Information on GO entries enriched by differentially expressed genes in GSE60993.

\begin{tabular}{|c|c|c|c|}
\hline Category & ID & Item & $P$ adjust \\
\hline BP & GO:0042119 & Neutrophil activation & $1.41 E-05$ \\
\hline $\mathrm{BP}$ & GO:0045088 & Regulation of innate immune response & $6.17 E-05$ \\
\hline $\mathrm{BP}$ & GO:0043312 & Neutrophil degranulation & $6.17 E-05$ \\
\hline $\mathrm{BP}$ & GO:0002283 & Neutrophil activation involved in immune response & $6.17 E-05$ \\
\hline BP & GO:0002446 & Neutrophil mediated immunity & $6.17 E-05$ \\
\hline BP & GO:0035743 & CD4-positive, alpha-beta T cell cytokine production & 0.000114 \\
\hline BP & GO:0045089 & Positive regulation of innate immune response & 0.000148 \\
\hline BP & GO:0002367 & Cytokine production involved in immune response & 0.000235 \\
\hline BP & GO:0098542 & Defense response to other organisms & 0.000496 \\
\hline BP & GO:0002369 & T cell cytokine production & 0.000501 \\
\hline BP & GO:0002720 & Positive regulation of cytokine production involved in immune response & 0.001009 \\
\hline BP & GO:0030595 & Leukocyte chemotaxis & 0.001785 \\
\hline BP & GO:0002440 & Production of molecular mediator of immune response & 0.001785 \\
\hline BP & GO:0002699 & Positive regulation of immune effector process & 0.001886 \\
\hline BP & GO:0070673 & Response to interleukin-18 & 0.001886 \\
\hline BP & GO:0002526 & Acute inflammatory response & 0.002469 \\
\hline BP & GO:0002690 & Positive regulation of leukocyte chemotaxis & 0.002469 \\
\hline BP & GO:0002718 & Regulation of cytokine production involved in immune response & 0.002508 \\
\hline $\mathrm{BP}$ & GO:0002456 & $\mathrm{T}$ cell mediated immunity & 0.002638 \\
\hline $\mathrm{BP}$ & GO:0002708 & Positive regulation of lymphocyte mediated immunity & 0.002638 \\
\hline $\mathrm{BP}$ & GO:0060337 & Type I interferon signaling pathway & 0.002638 \\
\hline $\mathrm{BP}$ & GO:0071357 & Cellular response to type I interferon & 0.002638 \\
\hline $\mathrm{BP}$ & GO:0042742 & Defense response to bacterium & 0.002638 \\
\hline BP & GO:0002702 & Positive regulation of production of molecular mediator of immune response & 0.002638 \\
\hline BP & GO:0034340 & Response to type I interferon & 0.002648 \\
\hline BP & GO:0060326 & Cell chemotaxis & 0.002648 \\
\hline BP & GO:0002726 & Positive regulation of $\mathrm{T}$ cell cytokine production & 0.003238 \\
\hline BP & GO:0002688 & Regulation of leukocyte chemotaxis & 0.003238 \\
\hline BP & GO:0002705 & Positive regulation of leukocyte mediated immunity & 0.004302 \\
\hline BP & GO:0002687 & Positive regulation of leukocyte migration & 0.00459 \\
\hline $\mathrm{BP}$ & GO:0032103 & Positive regulation of response to external stimulus & 0.005129 \\
\hline $\mathrm{BP}$ & GO:0050921 & Positive regulation of chemotaxis & 0.005298 \\
\hline BP & GO:0009615 & Response to virus & 0.005298 \\
\hline BP & GO:0002706 & Regulation of lymphocyte mediated immunity & 0.005706 \\
\hline BP & GO:0002700 & Regulation of production of molecular mediator of immune response & 0.005732 \\
\hline BP & GO:0070102 & Interleukin-6-mediated signaling pathway & 0.005732 \\
\hline BP & GO:0002724 & Regulation of $\mathrm{T}$ cell cytokine production & 0.006379 \\
\hline BP & GO:0002251 & Organ or tissue specific immune response & 0.008387 \\
\hline BP & GO:0002711 & Positive regulation of $\mathrm{T}$ cell mediated immunity & 0.009107 \\
\hline BP & GO:0097529 & Myeloid leukocyte migration & 0.009288 \\
\hline BP & GO:0019835 & Cytolysis & 0.009599 \\
\hline CC & GO:0042581 & Specific granule & $1.44 E-05$ \\
\hline $\mathrm{CC}$ & GO:0070820 & Tertiary granule & $1.44 E-05$ \\
\hline $\mathrm{CC}$ & GO:0030667 & Secretory granule membrane & 0.000164 \\
\hline $\mathrm{CC}$ & GO:0070821 & Tertiary granule membrane & 0.000654 \\
\hline $\mathrm{CC}$ & GO:0035579 & Specific granule membrane & 0.001009 \\
\hline $\mathrm{CC}$ & GO:0098802 & Plasma membrane receptor complex & 0.003883 \\
\hline CC & GO:1904724 & Tertiary granule lumen & 0.009249 \\
\hline
\end{tabular}


TABLE 5: Continued.

\begin{tabular}{lccc}
\hline Category & ID & Item & $P$ adjust \\
\hline CC & GO:0101003 & Ficolin-1-rich granule membrane & 0.009249 \\
CC & GO:0035580 & Specific granule lumen & 0.009249 \\
MF & GO:0004896 & Cytokine receptor activity & $6.26 E-05$ \\
MF & GO:0019955 & Cytokine binding & 0.003951 \\
\hline
\end{tabular}

reduce tissue damage and improve the therapeutic effect of cardiac remodeling [64]. Therefore, the therapeutic value and potential risk strategy of BDKRB2 agonist in AMI deserve further study.

As a neurotransmitter, 5-hydroxytryptamine (5-HT) not only plays a signal transduction role in immune cells but also regulates mood, behavior, and cardiovascular and gastrointestinal functions $[65,66]$. A study showed that 5-HT could regulate macrophage-mediated angiogenesis by reducing the expression of matrix metalloproteinase 12 in tumor-infiltrating macrophages [67]. In addition, it plays a role in the development of pulmonary hypertension depending on the expression of 5-hydroxytryptamine receptor 2B (HTR2B) in bone marrow progenitor cells [68]. de Las et al. showed that 5-HT could regulate macrophage polarization and maintain an anti-inflammatory state through 5-HT2B and 5-HT7 [69]. TACR3 is an important receptor protein, is expressed in the central nervous system, and activated the phosphatidylinositol-calcium second messenger system [70, 71]. In addition, the involvement of TACR3 in human reproductive systems such as normal hypogonadism is evident [72]. It has been reported that neurokinin B (endogenous ligand of TACR3) is involved in the pathogenesis of Parkinson's disease [73]. Schooling's findings suggest that drugs with TACR3 antagonists can regulate the reproductive axis to reduce cardiovascular morbidity and mortality [74]. Additionally, in our study, we observed the TACR3 upregulated in the AMI patients in comparison to UA controls. Gastrin-releasing peptide (GRP) is a neuropeptide whose receptor GRPR is expressed in a variety of cell types and can activate signaling pathways that induce neutrophil migration in vitro and in vivo [75]. In addition, GRPR was found to be expressed in immune cells [76]. Recent studies have shown that selective GRPR antagonist (RC-3095) has anti-inflammatory effects on arthritis and sepsis models [77, 78]. Interestingly, our research identified that HRH1, NPBWR1, BDKRB2, HTR2B, TACR3, BDKRB1, and GRPR are closely related to inflammation. This suggests that anti-inflammatory measures may be needed to treat AMI and UA.

The KEGG pathway enriched by the DEGs screened from the GSE60993 dataset is related to viral protein interaction with cytokine and cytokine receptor (hsa04061), human cytomegalovirus infection (hsa05163), and cytokinecytokine receptor interaction (hsa04060). Cytokines are extracellular molecules that transmit intercellular signals, mainly involved in cell differentiation and inflammation through binding to specific receptors on the cell surface
[79]. Inflammation plays an important role in different stages of coronary atherosclerosis, such as plaque formation, progression, stability, rupture, and acute thrombosis [80]. The combined action of neutrophils and mononuclear macrophages and inflammatory factors promotes the occurrence and development of atherosclerosis and acute coronary events [81]. It has been reported that some functions of mononuclear macrophages and neutrophils are closely related to many cytokines expressed on their surface and corresponding receptors, such as adhesion, chemotaxis, and phagocytosis [82, 83]. Many cytokines related to inflammation are involved in the development of ACS, such as TNF- $\alpha$ and IL-6 [84]. Boekholdt et al. showed that the increase of the IL- 8 level was associated with the increased risk of coronary artery disease [85]. Therefore, we speculate that the role of the cytokine-cytokine receptor interaction signaling pathway in the progression of AMI and UA may be achieved by regulating the inflammatory response.

In this study, the hub genes enriched in these 3 pathways are $\mathrm{C}-\mathrm{X}-\mathrm{C}$ chemokine receptor type 2 (CXCR2), the receptor of interleukin-8 (IL-8). Neutrophils play an important role in innate immunity [86]. CXC chemokines and related receptors have strong effects on chemotaxis and neutrophil activation, allowing them to migrate to infected or injured sites [87]. Yan et al. detected and analyzed the differential gene expression between AMI patients and stable angina patients and found that the mRNA expression related to CXCR2 in AMI patients was significantly upregulated compared with stable angina patients [88]. A number of studies have shown that the occurrence and development of coronary atherosclerotic heart disease are closely related to monocyte chemoattractant protein 1 (MCP-1), IL-8, and other major chemokines [89-91]. This suggests that the vital DEGs play essential roles in the development and progression of AMI and UA via different signaling pathways. Collectively, most of the DEGs were inflammation-related genes. Expression analysis and the correlation of key DEGs will undoubtedly aid in the understanding of the roles of such genes in the development of AMI and UA. Notably, in order to get more precise correlation reports, we need to conduct a series of experimental studies to prove.

However, some limitations of our study should be considered. For instance, the differential genes analyzed by the two chips did not overlap, which may be caused by various reasons such as the source of the included samples, the annotation analysis platform, and the time of blood sampling. In addition, this study is based on the analysis of chip data; further experiments are needed to verify. 


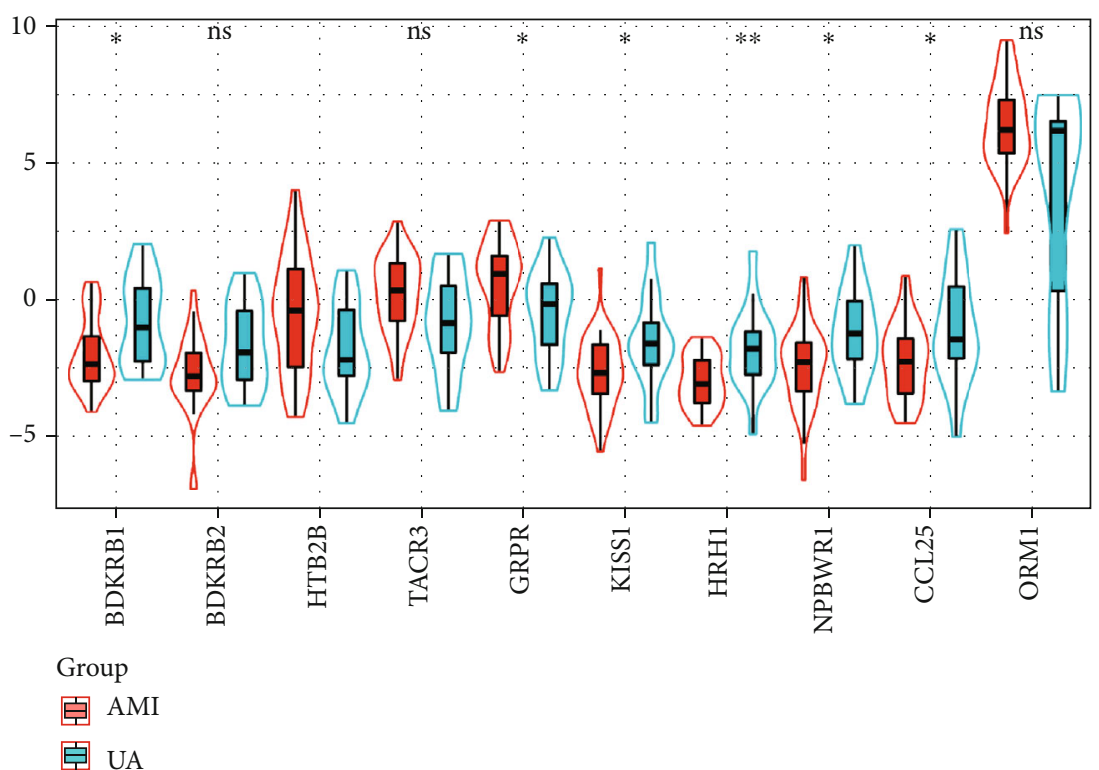

(a)

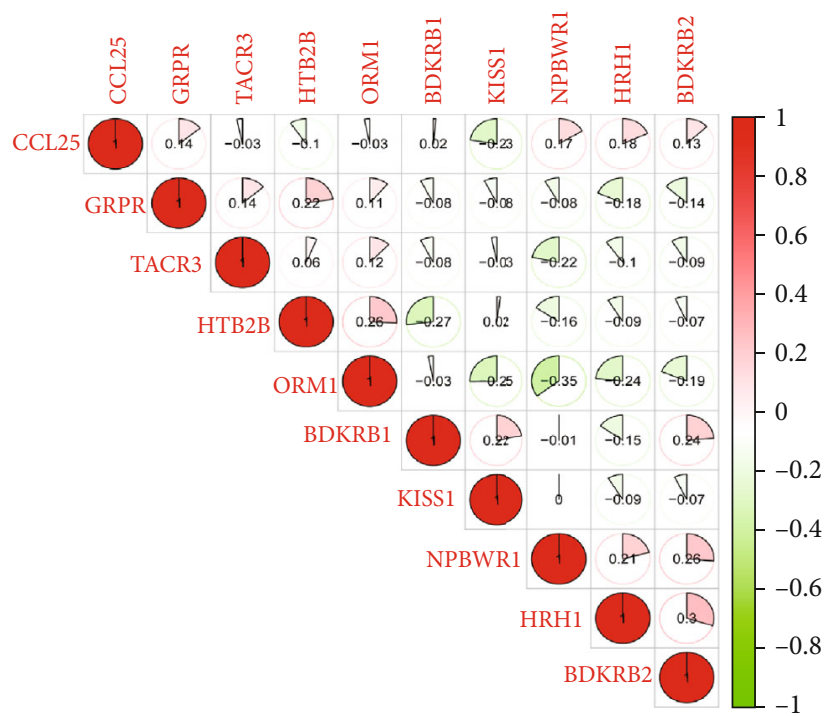

(b)

Figure 7: Continued. 


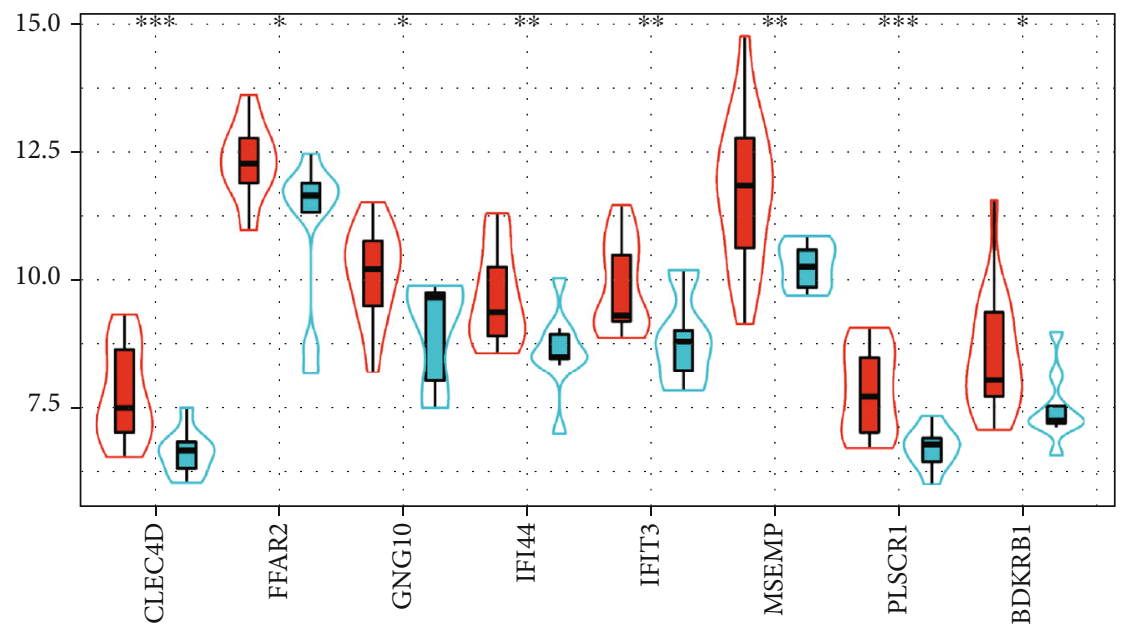

Group

追 AMI

官 UA

(c)

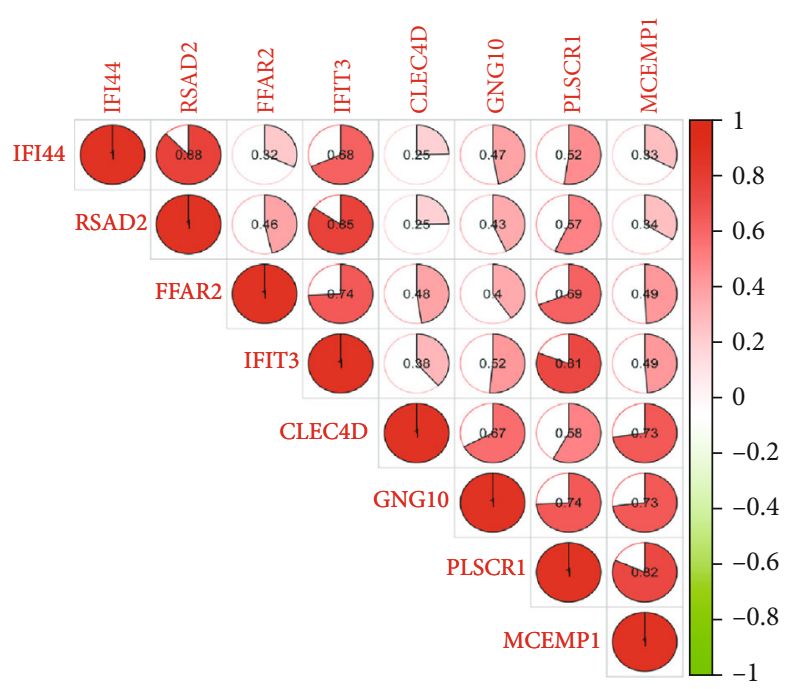

(d)

FIGURE 7: Expression level analysis of key genes in GSE29111 and GSE60993. (a) Expression analysis of GSE29111 key genes in the AMI and UA groups. (b) Bitmap for correlation analysis of key genes in GSE29111. (c) Expression analysis of GSE60993 key genes in the AMI and UA groups. (d) Bitmap for correlation analysis of key genes in GSE60993. ns: there is no statistically significant difference between groups. ${ }^{*} P<0.05 ;{ }^{* *} P<0.01 ;{ }^{* * *} P<0.001$.

\section{Conclusion}

In summary, the integrated bioinformatical analysis could improve our understanding of DEGs between AMI and UA. The integrated bioinformatical analysis could improve our understanding of DEGs between AMI and UA.

\section{Abbreviations}

5-HT: 5-Hydroxytryptamine

ACS: $\quad$ Acute coronary syndrome

AHA: American Heart Association
AMI: $\quad$ Acute myocardial infarction

BDKRB1: B1 bradykinin receptor

BDKRB2: B2 bradykinin receptor

BP: $\quad$ Biological process

CC: Cellular component

CCL25: C-C motif chemokine 25

CLEC4D: C-type lectin domain family 4 member D

CXCR2: $\quad$ C-X-C chemokine receptor type 2

DEGs: The differentially expressed genes

FC: $\quad$ Fold change

FFAR2: $\quad$ Free fatty acid receptor 2

FPR2: $\quad \mathrm{N}$-formyl peptide receptor 2 
GEO: Gene Expression Omnibus

GNG10: Guanine nucleotide-binding protein $\mathrm{G}(\mathrm{I}) / \mathrm{G}(\mathrm{S}) / \mathrm{G}(\mathrm{O})$ subunit gamma-10

GO: $\quad$ Gene Ontology

GRPR: Gastrin-releasing peptide receptor

HRH1: Histamine $\mathrm{H} 1$ receptor

HTR2B: 5-Hydroxytryptamine receptor 2B

IFI44: Interferon-induced protein 44

IFIT3: Interferon-induced protein with tetratricopeptide repeats 3

IL-1 $\alpha$ : Interleukin-1 alpha

IL-1 $\beta$ : Interleukin-1 beta

IL-6: Interleukin-6

IL-8: Interleukin-8

KEGG: $\quad$ Kyoto Encyclopedia of Genes and Genomes

KISS1: Metastasis-suppressor KiSS-1

MCC: $\quad$ Maximal Clique Centrality

MCEMP1: Mast cell-expressed membrane protein 1

MCODE: Molecular Complex Detection

MCP-1: $\quad$ Monocyte chemoattractant protein 1

MF: $\quad$ Molecular function

NCBI: The National Center for Biotechnology Information

NF- $\kappa$ B: $\quad$ Nuclear factor $-\kappa \mathrm{B}$

NIF-1: Hypoxia-inducible factor-1

NPB: $\quad$ Neuropeptide B

NPBWR1: Neuropeptide $\mathrm{B} / \mathrm{W}$ receptor type 1

NPBWR2: Neuropeptide $B / W$ receptor 2

NPW: Neuropeptide W

NSTEMI: Non-ST-segment elevation acute coronary syndrome

ORM1: $\quad$ Alpha-1-acid glycoprotein 1

PLSCR1: $\quad$ Phospholipid scramblase 1

PPI: $\quad$ Protein-protein interaction

ROS: $\quad$ Reactive oxygen species

RSAD2: Radical S-adenosyl methionine domaincontaining protein 2

STAT3: Signal transduction and transcription activation factor 3

STEMI: ST-segment elevation acute myocardial infarction

TACR3: Neuromedin-K receptor

TCGA: The Cancer Genome Atlas

TNF- $\alpha$ : Tumor necrosis factor- $\alpha$

UA: Unstable angina.

\section{Data Availability}

The raw data supporting the conclusions of this article will be made available by the authors, without undue reservation. All data obtained or analyzed during this study are available from the published article and its supplementary information files. The datasets during the current study are available from the corresponding author upon reasonable request.

\section{Conflicts of Interest}

The authors declare no competing interests in any respect.

\section{Authors' Contributions}

SG and JW conceived and designed the study. XZ and MW provided significative suggestions on the methodology. $\mathrm{ZH}$, $\mathrm{CW}$, and SL collected the data. XL, JZ, and SJ performed the data analysis; SG and JW wrote and revised the manuscript. All authors were responsible for reviewing the data. All authors read and approved the final manuscript.

\section{Acknowledgments}

This work was supported by the Young Scientists Training Program of Beijing University of Chinese Medicine and the National Nature Science Foundation of China (Grant no. 82074284 ).

\section{Supplementary Materials}

Supplementary 1. Supplementary Table 1: information of differentially expressed genes in GSE29111.

Supplementary 2. Supplementary Table 2: information of differentially expressed genes in GSE60993.

\section{References}

[1] E. Tahto, R. Jadric, L. Pojskic, and E. Kicic, "Neutrophil-tolymphocyte ratio and its relation with markers of inflammation and myocardial necrosis in patients with acute coronary syndrome," Medical Archives, vol. 71, no. 5, pp. 312-315, 2017.

[2] K. A. Thygesen and J. S. Alpert, "The definitions of acute coronary syndrome, myocardial infarction, and unstable angina," Current Cardiology Reports, vol. 3, no. 4, pp. 268-272, 2001.

[3] F. Bouisset, J. B. Ruidavets, J. Dallongeville et al., "Comparison of short- and long-term prognosis between ST-elevation and non-ST-elevation myocardial infarction," Journal of Clinical Medicine, vol. 10, no. 2, p. 180, 2021.

[4] S. S. Virani, A. Alonso, E. J. Benjamin et al., "Heart disease and stroke statistics-2020 update: a report from the American Heart Association," Circulation, vol. 141, no. 9, pp. e139e596, 2020.

[5] Y. Cui, K. Hao, J. Takahashi et al., "Age-specific trends in the incidence and in-hospital mortality of acute myocardial infarction over 30 years in Japan- report from the Miyagi AMI Registry study," Circulation Journal, vol. 81, no. 4, pp. 520-528, 2017.

[6] A. W. Ibrahim, T. C. Riddell, and C. M. Devireddy, "Acute myocardial infarction," Critical Care Clinics, vol. 30, no. 3, pp. 341-364, 2014.

[7] N. Rajpurohit, S. Z. Ayaz, J. Yee, M. A. Khan, and A. Stys, "Review of acute coronary syndromes: diagnosis and management of unstable angina and non ST-elevation myocardial infarction," SD Med, vol. 68, no. 2, 2015.

[8] A. Yaghoubi, Z. Golmohamadi, A. Alizadehasl, and R. Azarfarin, "Role of platelet parameters and haematological indices in myocardial infarction and unstable angina," Journal of the Pakistan Medical Association, vol. 63, no. 9, pp. 11331137, 2013.

[9] U. Thadani and T. L. Ripley, "Side effects of using nitrates to treat heart failure and the acute coronary syndromes, unstable angina and acute myocardial infarction," Expert Opinion on Drug Safety, vol. 6, no. 4, pp. 385-396, 2007. 
[10] J. Shin, J. E. Edelberg, and M. K. Hong, "Vulnerable atherosclerotic plaque: clinical implications," Current Vascular Pharmacology, vol. 1, no. 2, pp. 183-204, 2003.

[11] A. S. Go, C. Iribarren, M. Chandra et al., "Statin and betablocker therapy and the initial presentation of coronary heart disease," Annals of Internal Medicine, vol. 144, no. 4, pp. 229-238, 2006.

[12] S. Udhaya Kumar, D. Thirumal Kumar, R. Bithia et al., "Analysis of differentially expressed genes and molecular pathways in familial hypercholesterolemia involved in atherosclerosis: a systematic and bioinformatics approach," Frontiers in Genetics, vol. 11, p. 734, 2020.

[13] J. Wan, S. Jiang, Y. Jiang et al., "Data mining and expression analysis of differential lncRNA ADAMTS9-AS1 in prostate cancer," Frontiers in Genetics, vol. 10, p. 1377, 2020.

[14] S. Udhaya Kumar, B. Rajan, D. Thirumal Kumar et al., "Involvement of essential signaling cascades and analysis of gene networks in diabesity," Genes, vol. 11, no. 11, p. 1256, 2020.

[15] D. Fu, B. Zhang, L. Yang, S. Huang, and W. Xin, “Development of an immune-related risk signature for predicting prognosis in lung squamous cell carcinoma," Frontiers in Genetics, vol. 11, p. 978, 2020.

[16] G. Russo, C. Zegar, and A. Giordano, "Advantages and limitations of microarray technology in human cancer," Oncogene, vol. 22, no. 42, pp. 6497-6507, 2003.

[17] Z. Liu, C. Ma, J. Gu, and M. Yu, "Potential biomarkers of acute myocardial infarction based on weighted gene co-expression network analysis," BioMedical Engineering OnLine, vol. 18, no. 1, p. 9, 2019.

[18] Q. Wang, B. Liu, Y. Wang, B. Bai, T. Yu, and X. M. Chu, "The biomarkers of key miRNAs and target genes associated with acute myocardial infarction," PeerJ, vol. 8, article e9129, 2020.

[19] T. Barrett, S. E. Wilhite, P. Ledoux et al., "NCBI GEO: archive for functional genomics data sets-update," Nucleic Acids Research, vol. 41, no. D1, pp. D991-D995, 2012.

[20] M. E. Ritchie, B. Phipson, D. Wu et al., "limma powers differential expression analyses for RNA-sequencing and microarray studies," Nucleic Acids Research, vol. 43, no. 7, article e47, 2015.

[21] D. Szklarczyk, A. L. Gable, D. Lyon et al., "STRING v11: protein-protein association networks with increased coverage, supporting functional discovery in genome-wide experimental datasets," Nucleic Acids Research, vol. 47, no. D1, pp. D607D613, 2019.

[22] P. Shannon, A. Markiel, O. Ozier et al., "Cytoscape: a software environment for integrated models of biomolecular interaction networks," Genome Research, vol. 13, no. 11, pp. 24982504, 2003.

[23] S. Shen, J. Kong, Y. Qiu, X. Yang, W. Wang, and L. Yan, "Identification of core genes and outcomes in hepatocellular carcinoma by bioinformatics analysis," Journal of Cellular Biochemistry, vol. 120, no. 6, pp. 10069-10081, 2019.

[24] C. H. Chin, S. H. Chen, H. H. Wu, C. W. Ho, M. T. Ko, and C. Y. Lin, "cytoHubba: identifying hub objects and subnetworks from complex interactome," BMC Systems Biology, vol. 8, article S11, Supplement 4, 2014.

[25] P. D. Thomas, "The Gene Ontology and the meaning of biological function," Methods in Molecular Biology, vol. 1446, pp. 15-24, 2017.
[26] M. Kanehisa, M. Furumichi, M. Tanabe, Y. Sato, and K. Morishima, "KEGG: new perspectives on genomes, pathways, diseases and drugs," Nucleic Acids Research, vol. 45, no. D1, pp. D353-D361, 2017.

[27] G. Yu, L. G. Wang, Y. Han, and Q. Y. He, “clusterProfiler: an R package for comparing biological themes among gene clusters," OMICS: A Journal of Integrative Biology, vol. 16, no. 5, pp. 284-287, 2012.

[28] W. Walter, F. Sanchez-Cabo, and M. Ricote, "GOplot: an R package for visually combining expression data with functional analysis," Bioinformatics, vol. 31, no. 17, pp. 2912-2914, 2015.

[29] Z. Gu, R. Eils, and M. Schlesner, "Complex heatmaps reveal patterns and correlations in multidimensional genomic data," Bioinformatics, vol. 32, no. 18, pp. 2847-2849, 2016.

[30] L. Pei, J. Li, Z. Xu, N. Chen, X. Wu, and J. Chen, "Effect of high hydrostatic pressure on aroma components, amino acids, and fatty acids of Hami melon (Cucumis melo L. var. reticulatus naud.) juice," Food Science \& Nutrition, vol. 8, no. 3, pp. 1394-1405, 2020.

[31] K. R. Kawamoto, M. B. Davis, and C. S. Duvernoy, "Acute coronary syndromes: differences in men and women," Current Atherosclerosis Reports, vol. 18, no. 12, p. 73, 2016.

[32] S. Johnston and A. L. Eckhardt, "Fatigue and acute coronary syndrome: a systematic review of contributing factors," Heart \& Lung, vol. 47, no. 3, pp. 192-204, 2018.

[33] O. Bebb, F. G. Smith, A. Clegg, M. Hall, and C. P. Gale, "Frailty and acute coronary syndrome: a structured literature review," European Heart Journal Acute Cardiovascular Care, vol. 7, no. 2, pp. 166-175, 2018.

[34] Z. Xu, Y. Zhou, Y. Cao, T. L. Dinh, J. Wan, and M. Zhao, "Identification of candidate biomarkers and analysis of prognostic values in ovarian cancer by integrated bioinformatics analysis," Medical Oncology, vol. 33, no. 11, p. 130, 2016.

[35] X. Guo, Y. Wang, C. Wang, and J. Chen, "Identification of several hub-genes associated with periodontitis using integrated microarray analysis," Molecular Medicine Reports, vol. 11, no. 4, pp. 2541-2547, 2015.

[36] E. Golia, G. Limongelli, F. Natale et al., "Inflammation and cardiovascular disease: from pathogenesis to therapeutic target," Current Atherosclerosis Reports, vol. 16, no. 9, p. 435, 2014.

[37] D. Tousoulis, C. Psarros, M. Demosthenous, R. Patel, C. Antoniades, and C. Stefanadis, "Innate and adaptive inflammation as a therapeutic target in vascular disease: the emerging role of statins," Journal of the American College of Cardiology, vol. 63, no. 23, pp. 2491-2502, 2014.

[38] F. Roubille, D. Busseuil, N. Merlet, E. A. Kritikou, E. Rheaume, and J. C. Tardif, "Investigational drugs targeting cardiac fibrosis," Expert Review of Cardiovascular Therapy, vol. 12, no. 1, pp. 111-125, 2014.

[39] S. B. Ong, S. Hernández-Reséndiz, G. E. Crespo-Avilan et al., "Inflammation following acute myocardial infarction: multiple players, dynamic roles, and novel therapeutic opportunities," Pharmacology \& Therapeutics, vol. 186, pp. 73-87, 2018.

[40] L. Timmers, G. Pasterkamp, V. C. de Hoog, F. Arslan, Y. Appelman, and D. P. de Kleijn, "The innate immune response in reperfused myocardium," Cardiovascular Research, vol. 94, no. 2, pp. 276-283, 2012.

[41] A. Abbate, F. N. Salloum, E. Vecile et al., "Anakinra, a recombinant human interleukin-1 receptor antagonist, inhibits apoptosis in experimental acute myocardial infarction," Circulation, vol. 117, no. 20, pp. 2670-2683, 2008. 
[42] H. Bai, K. Sun, J. H. Wu et al., "Proteomic and metabolomic characterization of cardiac tissue in acute myocardial ischemia injury rats," PLoS One, vol. 15, no. 5, article e231797, p. e0231797, 2020.

[43] T. Kishi, "Heart failure as an autonomic nervous system dysfunction," Journal of Cardiology, vol. 59, no. 2, pp. 117-122, 2012.

[44] M. Vaseghi and K. Shivkumar, "The role of the autonomic nervous system in sudden cardiac death," Progress in Cardiovascular Diseases, vol. 50, no. 6, pp. 404-419, 2008.

[45] K. Shinlapawittayatorn, K. Chinda, S. Palee et al., "Low-amplitude, left vagus nerve stimulation significantly attenuates ventricular dysfunction and infarct size through prevention of mitochondrial dysfunction during acute ischemiareperfusion injury," Heart Rhythm, vol. 10, no. 11, pp. 17001707, 2013.

[46] K. Shinlapawittayatorn, K. Chinda, S. Palee et al., "Vagus nerve stimulation initiated late during ischemia, but not reperfusion, exerts cardioprotection via amelioration of cardiac mitochondrial dysfunction," Heart Rhythm, vol. 11, no. 12, pp. 22782287, 2014.

[47] J. Wang, J. Cheng, C. Zhang, and X. Li, "Cardioprotection effects of sevoflurane by regulating the pathway of neuroactive ligand-receptor interaction in patients undergoing coronary artery bypass graft surgery," Computational and Mathematical Methods in Medicine, vol. 2017, Article ID 3618213, 7 pages, 2017.

[48] P. Chen, B. Long, Y. Xu, W. Wu, and S. Zhang, "Identification of crucial genes and pathways in human arrhythmogenic right ventricular cardiomyopathy by coexpression analysis," Frontiers in Physiology, vol. 9, p. 1778, 2018.

[49] S. Z. Guo and W. J. Liu, "Constructing differential coexpression network to predict key pathways for myocardial infarction," Experimental and Therapeutic Medicine, vol. 17, no. 4, pp. 3029-3034, 2019.

[50] M. Ruiz-Meana, D. Garcia-Dorado, M. Juliá et al., "Protective effect of HOE642, a selective blocker of $\mathrm{Na}^{+}-\mathrm{H}^{+}$exchange, against the development of rigor contracture in rat ventricular myocytes," Experimental Physiology, vol. 85, no. 1, pp. 17-25, 2000.

[51] D. Garcia-Dorado, M. Ruiz-Meana, J. Inserte, A. RodriguezSinovas, and H. M. Piper, "Calcium-mediated cell death during myocardial reperfusion," Cardiovascular Research, vol. 94, no. 2, pp. 168-180, 2012.

[52] T. Numata, K. Takahashi, and R. Inoue, “"TRP inflammation" relationship in cardiovascular system," Seminars in Immunopathology, vol. 38, no. 3, pp. 339-356, 2016.

[53] C. A. Akdis and F. E. Simons, "Histamine receptors are hot in immunopharmacology," European Journal of Pharmacology, vol. 533, no. 1-3, pp. 69-76, 2006.

[54] S. K. Ganesh, V. Tragante, W. Guo et al., "Loci influencing blood pressure identified using a cardiovascular gene-centric array," Human Molecular Genetics, vol. 22, no. 8, pp. 16631678, 2013.

[55] M. E. Bhuiyan, H. Waki, S. S. Gouraud, M. Takagishi, A. Kohsaka, and M. Maeda, "Histamine receptor H1 in the nucleus tractus solitarii regulates arterial pressure and heart rate in rats," American Journal of Physiology. Heart and Circulatory Physiology, vol. 301, no. 2, pp. H523-H529, 2011.

[56] I. Rozenberg, S. H. Sluka, L. Rohrer et al., "Histamine H1 receptor promotes atherosclerotic lesion formation by increas- ing vascular permeability for low-density lipoproteins," Arteriosclerosis, Thrombosis, and Vascular Biology, vol. 30, no. 5, pp. 923-930, 2010.

[57] R. Fujii, H. Yoshida, S. Fukusumi et al., "Identification of a Neuropeptide Modified with Bromine as an Endogenous Ligand for GPR7," Journal of Biological Chemistry, vol. 277, no. 37, pp. 34010-34016, 2002.

[58] S. Brezillon, V. Lannoy, J. D. Franssen et al., "Identification of Natural Ligands for the Orphan G Protein-coupled Receptors GPR7 and GPR8," Journal of Biological Chemistry, vol. 278, no. 2, pp. 776-783, 2003.

[59] D. M. Chottova, "Distribution and function of neuropeptides W/B signaling system," Frontiers in Physiology, vol. 9, p. 981, 2018.

[60] G. Singh and A. P. Davenport, "Neuropeptide B and W: neurotransmitters in an emerging G-protein-coupled receptor system," British Journal of Pharmacology, vol. 148, no. 8, pp. 1033-1041, 2006.

[61] L. Ji, H. Zhu, H. Chen et al., "Modulation of CaV1.2 calcium channel by neuropeptide $\mathrm{W}$ regulates vascular myogenic tone via G protein-coupled receptor 7," Journal of Hypertension, vol. 33, no. 12, pp. 2431-2442, 2015.

[62] N. Yu, C. Chu, T. Kunitake, K. Kato, M. Nakazato, and H. Kannan, "Cardiovascular actions of central neuropeptide W in conscious rats," Regulatory Peptides, vol. 138, no. 2-3, pp. 82-86, 2007.

[63] A. J. Manolis, M. E. Marketou, I. Gavras, and H. Gavras, "Cardioprotective properties of bradykinin: role of the $\mathrm{B}_{2}$ receptor," Hypertension Research, vol. 33, no. 8, pp. 772$777,2010$.

[64] M. Marketou, E. Kintsurashvili, K. N. Papanicolaou, H. A. Lucero, I. Gavras, and H. Gavras, "Cardioprotective effects of a selective $\mathrm{B}_{2}$ receptor agonist of bradykinin post-acute myocardial infarct," American Journal of Hypertension, vol. 23, no. 5, pp. 562-568, 2010.

[65] G. P. Ahern, "5-HT and the immune system," Current Opinion in Pharmacology, vol. 11, no. 1, pp. 29-33, 2011.

[66] M. Lesurtel, C. Soll, R. Graf, and P. A. Clavien, "Role of serotonin in the hepato-gastrointestinal tract: an old molecule for new perspectives," Cellular and Molecular Life Sciences, vol. 65, no. 6, pp. 940-952, 2008.

[67] A. Nocito, F. Dahm, W. Jochum et al., "Serotonin regulates macrophage-mediated angiogenesis in a mouse model of colon cancer allografts," Cancer Research, vol. 68, no. 13, pp. 51525158, 2008.

[68] J. M. Launay, P. Hervé, J. Callebert et al., "Serotonin 5-HT2B receptors are required for bone-marrow contribution to pulmonary arterial hypertension," Blood, vol. 119, no. 7, pp. 1772-1780, 2012.

[69] M. de las Casas-Engel, A. Domínguez-Soto, E. Sierra-Filardi et al., "Serotonin skews human macrophage polarization through HTR2B and HTR7," Journal of Immunology, vol. 190, no. 5, pp. 2301-2310, 2013.

[70] X. Langlois, C. Wintmolders, P. te Riele, J. E. Leysen, and M. Jurzak, "Detailed distribution of neurokinin 3 receptors in the rat, guinea pig and gerbil brain: a comparative autoradiographic study," Neuropharmacology, vol. 40, no. 2, pp. 242253, 2001.

[71] K. Obata, T. Shimo, T. Okui et al., “Tachykinin receptor 3 distribution in human oral squamous cell carcinoma," Anticancer Research, vol. 36, no. 12, pp. 6335-6342, 2016. 
[72] A. K. Topaloglu, F. Reimann, M. Guclu et al., "TAC3 and TACR3 mutations in familial hypogonadotropic hypogonadism reveal a key role for Neurokinin B in the central control of reproduction," Nature Genetics, vol. 41, no. 3, pp. 354$358,2009$.

[73] V. Mesnage, J. L. Houeto, A. M. Bonnet et al., "Neurokinin B, neurotensin, and cannabinoid receptor antagonists and Parkinson disease," Clinical Neuropharmacology, vol. 27, no. 3, pp. 108-110, 2004.

[74] C. M. Schooling, "Tachykinin neurokinin 3 receptor antagonists: a new treatment for cardiovascular disease?," The Lancet, vol. 390, no. 10095, pp. 709-711, 2017.

[75] R. S. Czepielewski, B. N. Porto, L. B. Rizzo et al., "Gastrinreleasing peptide receptor (GRPR) mediates chemotaxis in neutrophils," Proceedings of the National Academy of Sciences of the United States of America, vol. 109, no. 2, pp. 547-552, 2012.

[76] S. Zhou, E. N. Potts, F. Cuttitta, W. M. Foster, and M. E. Sunday, "Gastrin-releasing peptide blockade as a broad-spectrum anti-inflammatory therapy for asthma," Proceedings of the National Academy of Sciences of the United States of America, vol. 108, no. 5, pp. 2100-2105, 2011.

[77] P. G. Oliveira, C. V. Brenol, M. I. Edelweiss et al., "Effects of an antagonist of the bombesin/gastrin-releasing peptide receptor on complete Freund's adjuvant-induced arthritis in rats," Peptides, vol. 29, no. 10, pp. 1726-1731, 2008.

[78] F. Dal-Pizzol, L. P. di Leone, C. Ritter et al., "Gastrin-releasing peptide receptor antagonist effects on an animal model of sepsis," American Journal of Respiratory and Critical Care Medicine, vol. 173, no. 1, pp. 84-90, 2006.

[79] K. Ozaki and W. J. Leonard, "Cytokine and Cytokine Receptor Pleiotropy and Redundancy," Journal of Biological Chemistry, vol. 277, no. 33, pp. 29355-29358, 2002.

[80] P. Libby, "Inflammation in atherosclerosis," Arteriosclerosis, Thrombosis, and Vascular Biology, vol. 32, no. 9, pp. 20452051, 2012.

[81] B. Legein, L. Temmerman, E. A. Biessen, and E. Lutgens, "Inflammation and immune system interactions in atherosclerosis," Cellular and Molecular Life Sciences, vol. 70, no. 20, pp. 3847-3869, 2013.

[82] C. J. Thomas and K. Schroder, "Pattern recognition receptor function in neutrophils," Trends in Immunology, vol. 34, no. 7, pp. 317-328, 2013.

[83] L. Xing and D. G. Remick, "Relative cytokine and cytokine inhibitor production by mononuclear cells and neutrophils," Shock, vol. 20, no. 1, pp. 10-16, 2003.

[84] K. Mourouzis, E. Oikonomou, G. Siasos et al., "Pro-inflammatory cytokines in acute coronary syndromes," Current Pharmaceutical Design, vol. 26, no. 36, pp. 4624-4647, 2020.

[85] S. M. Boekholdt, R. J. Peters, C. E. Hack et al., "IL-8 plasma concentrations and the risk of future coronary artery disease in apparently healthy men and Women," Arteriosclerosis, Thrombosis, and Vascular Biology, vol. 24, no. 8, pp. 15031508, 2004.

[86] S. de Oliveira, C. C. Reyes-Aldasoro, S. Candel, S. A. Renshaw, V. Mulero, and A. Calado, "Cxcl8 (IL-8) mediates neutrophil recruitment and behavior in the zebrafish inflammatory response," Journal of Immunology, vol. 190, no. 8, pp. 43494359, 2013.

[87] A. M. Ritzman, J. M. Hughes-Hanks, V. A. Blaho, L. E. Wax, W. J. Mitchell, and C. R. Brown, "The chemokine receptor
CXCR2 ligand KC (CXCL1) mediates neutrophil recruitment and is critical for development of experimental Lyme arthritis and carditis," Infection and Immunity, vol. 78, no. 11, pp. 4593-4600, 2010.

[88] W. Yan, S. Wen, L. Wang, Q. Duan, and L. Ding, "Comparison of cytokine expressions in acute myocardial infarction and stable angina stages of coronary artery disease," International Journal of Clinical and Experimental Medicine, vol. 8, no. 10, pp. 18082-18089, 2015.

[89] C. R. Li, L. M. Wang, Z. Gong et al., "Expression characteristics of neutrophil and mononuclear-phagocyte related genes mRNA in the stable angina pectoris and acute myocardial infarction stages of coronary artery disease," Journal of Geriatric Cardiology, vol. 12, no. 3, pp. 279-286, 2015.

[90] M. Vistnes, "Macrophage inflammatory Protein-1 $\beta$ : a novel prognostic biomarker in atherosclerosis?," Cardiology, vol. 121, no. 3, pp. 149-151, 2012.

[91] C. Papadopoulou, V. Corrigall, P. R. Taylor, and R. N. Poston, "The role of the chemokines MCP-1, GRO- $\alpha$, IL- 8 and their receptors in the adhesion of monocytic cells to human atherosclerotic plaques," Cytokine, vol. 43, no. 2, pp. 181-186, 2008. 